\title{
EI terremoto de Lorca (2011) en el contexto de la peligrosidad y el riesgo sísmico en Murcia
}

\author{
BELÉN BENITO OTERINO ${ }^{1}$, ALICIA RIVAS MEDINA ${ }^{1}$, \\ JORGE M. GASPAR-ESCRIBANO ${ }^{1}$ \& PATRICK MURPHY ${ }^{2}$ \\ ${ }^{1}$ ETSI Topografía, Geodesia y Cartografía. Universidad Politécnica de Madrid. \\ ${ }^{2}$ Broadway Malyan, Madrid. \\ mariabelen.benito@upm.es
}

\begin{abstract}
Resumen
Este trabajo presenta un análisis de diferentes aspectos relacionados con el sismo principal del 11 de Mayo de 2011, con epicentro en las proximidades de Lorca, que abarcan desde el movimiento fuerte registrado hasta el daño observado en diferentes tipologías constructivas, contrastando todo ello con los resultados de estudios previos de peligrosidad y riesgo sísmico en la provincia de Murcia. La cuestión esencial que se plantea en el análisis es si tanto el movimiento como el daño entraban dentro de lo esperado o pueden considerarse anómalos en el marco del riesgo sísmico del sureste de España. A este respecto se hacen una serie de reflexiones y se extraen lecciones aprendidas del terremoto, que llevan a proponer recomendaciones de cara a la revisión de la Norma Sismorresistente Española, así como a definir medidas para la reducción del riesgo sísmico en la región.
\end{abstract}

Palabras clave: peligrosidad sísmica, movimiento fuerte, riesgo sísmico, vulnerabilidad.

\section{The 2011 Lorca earthquake in the context of seismic hazard and risk in} Murcia

\begin{abstract}
An analysis of the different aspects related to the May 11th, 2011 Lorca earthquake is presented, covering recorded ground motions, damage observed in different building typologies, and contrasting these observations with previous results on seismic hazard and seismic risk obtained in the province of Murcia. The essential question addressed in the analysis is whether observed ground motions and physical damage can be considered as expected or as anomalous in the frame of seismic risk in southeastern Spain. In this respect, a number of reflections are carried out and several learned lessons from the earthquake are extracted, which leads to the proposal of different recommendations for the future revision of the Spanish earthquake-resistant provisions, as well as for defining risk reduction measurements in the region.
\end{abstract}

Keywords: seismic hazard, strong ground motion, seismic risk, vulnerability.

\section{Referencia normalizada}

Benito Oterino, B., Rivas Medina, A.; Gaspar-Escribano, J. M., Murphy, P. (2012). El terremoto de Lorca (2011) en el contexto de la peligrosidad y el riesgo sísmico en Murcia. Física de la Tierra, Vol. 24, 255-287.

Sumario: Introducción 1. Peligrosidad sísmica en Lorca. 1.1. Sismicidad histórica e instrumental en la provincia de Murcia. 1.2. Movimiento fuerte registrado en Lorca 2011 y predicho en análogas condiciones. 1.2.1. Simulación de aceleraciones pico, PGA. 1.2.2. Simulación de espectros de respuesta. 1.2.3. Desagregación: sismos de control. 1.3. Conclusiones sobre el movimiento registrado en el marco de la peligrosidad. 2. Riesgo sísmico en Lorca y daño observado 2.1. El riesgo de Lorca enmarcado en el riesgo de la provincia de Murcia. 2.2. Lorca: Población con prioridad de actuación según los resultados del Proyecto RISMUR. 2.3. Daños observados después del terremoto. 
2.3.1. Triaje técnico de edificios. 2.3.2. Datos de daños. 2.3.3. Conclusiones referentes al riesgo sísmico en Lorca y los daños observados en 2011. 3. Discusión final y lecciones aprendidas. Referencias bibliográficas

\section{Introducción}

El terremoto de Lorca es el terremoto más importante ocurrido en las últimas décadas en España, no solo porque ha causado víctimas y graves daños, sino porque ha arrojado una importante cantidad de datos que permiten estudiarlo. En este trabajo nos centramos en los estudios de peligrosidad y riesgo sísmico desarrollados en Lorca y, de modo más general, en la Región de Murcia en los últimos años.

\section{Peligrosidad sísmica en Lorca}

La peligrosidad sísmica en Lorca se puede calificar de alta, en el contexto de la Península Ibérica, si bien es moderada en un contexto mundial. Dicha peligrosidad se explica por la localización de la población en la traza del sistema de fallas activas de Alhama de Murcia, con ocurrencia de terremotos significativos en el pasado. Además, se han propuesto procesos de disparo entre series sísmicas relativamente cercanas en el espacio (Benito et al. 2007), que parecen confirmarse también por la proximidad temporal de dichas series (Mula, 1999; Bullas, 2002, La Paca, 2005 y Lorca, 2011). Este hecho, unido al daño observado por terremotos de bajamoderada magnitud, confiere un riesgo sísmico relevante a la población.

Los diversos estudios de peligrosidad desarrollados hasta la fecha proporcionan resultados de aceleración pico PGA en roca para la probabilidad de excedencia del $10 \%$ en 50 años, empleada en el diseño de viviendas convencionales, en un rango de variación entre 0.12 g y 0.24 g (Buforn et al. 2005; GarcíaMayordomo, 2005; Benito et al., 2006; García-Mayordomo et al. 2007; García Blanco 2009; Crespo 2011), estando los de la norma Española NCSE-02 en el límite inferior. Ninguno de estos estudios ha considerado las fallas como unidades independientes (con peso relevante) en el cálculo de la peligrosidad; hecho que se traduce en isolíneas del movimiento esperado que no correlacionan específicamente con la geometría de las fallas, distribuyéndose la peligrosidad en zonas más amplias.

En la Figura 1 se muestra el mapa de la norma sismorresistente Española, NCSE-02. El parámetro representado en el mapa es la aceleración básica, $\mathrm{a}_{\mathrm{b}}$, correspondiente a la aceleración pico horizontal PGA en suelo duro para probabilidades de excedencia de $10 \%$ en 50 años. Los valores esperados en la provincia de Murcia oscilan entre $0.04 \mathrm{~g}$ y $0.16 \mathrm{~g}$, siendo el valor dado en Lorca de $0.12 \mathrm{~g}$. Ese valor fue excedido en la componente $\mathrm{N} 30 \mathrm{~W}$ por un factor 3 durante el terremoto principal de 2011, alcanzando dicha componente un valor de $0.37 \mathrm{~g}$. No obstante la media geométrica de la aceleración pico horizontal, considerando las dos componentes horizontales, fue de $0.21 \mathrm{~g}$, es decir, prácticamente doble de la dada en el mapa de la NCSE-02. 


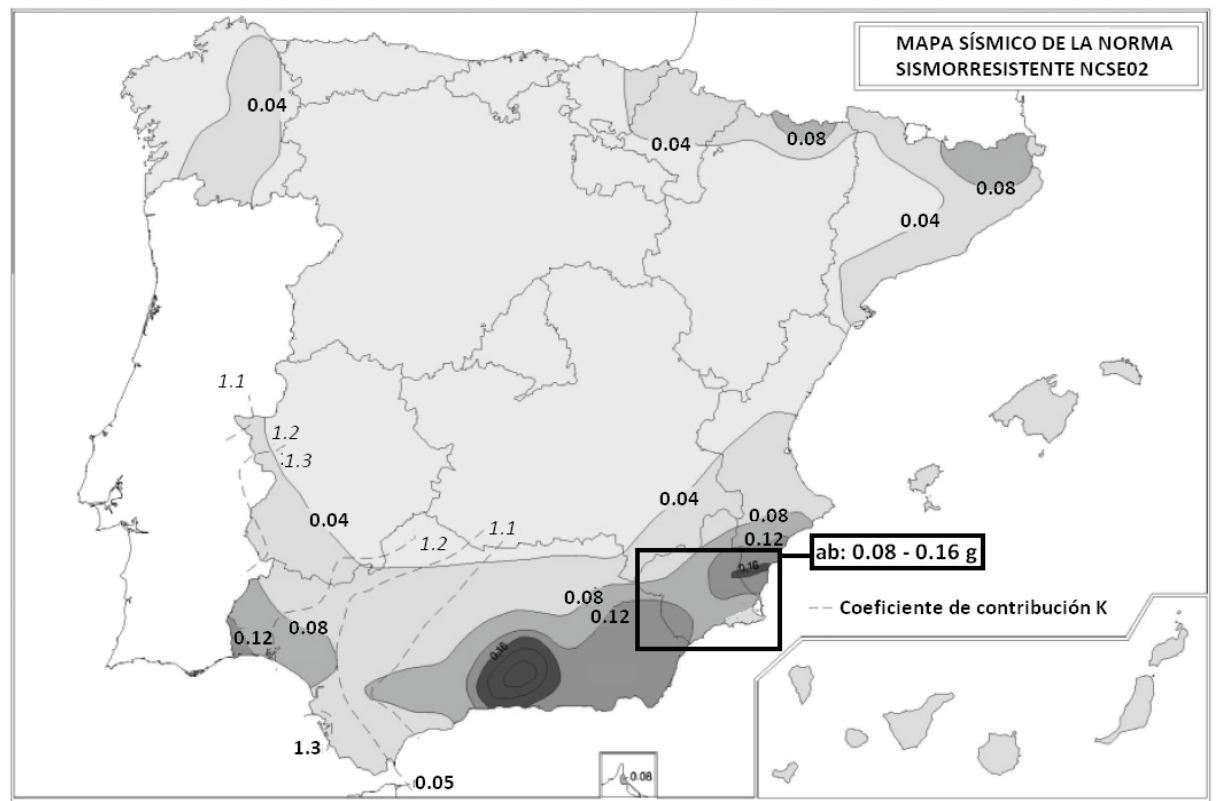

Fig. 1. Mapa de aceleración básica para período de retorno de 475 años de la Norma de Construcción Sismorresistente Española NCSE-02, destacando en el recuadro la peligrosidad de la provincia de Murcia.

Cabe preguntarse si la peligrosidad de Lorca está infraestimada en el mapa de la NCSE-02 o bien el valor de aceleración registrado en 2011 corresponde justo a ese $10 \%$ de probabilidad de excedencia en 50 años que se admite en la normativa para diseño de viviendas convencionales. A este respecto acudimos a otro estudio de peligrosidad en la provincia de Murcia, integrado en el proyecto RISMUR destinado a evaluar el riesgo sísmico en la región- que fue la base del plan de emergencias SISMIMUR activado precisamente en el terremoto de Lorca 2011. Ese estudio concluyó, en la parte de peligrosidad, con el mapa de la Figura 2a que muestra la PGA horizontal en roca para periodo de retorno de 475 años, equivalente también a probabilidad de excedencia del $10 \%$ en 50 años. El estudio siguió una metodología probabilista zonificada, en la línea metodológica PSHA (Probabilistic Seismic Hazard Assessment), adoptando un árbol lógico con nodos que contemplan diferentes opciones de zonificación y ecuaciones de predicción del movimiento fuerte (GMPE's). En este caso el estudio se desarrolló directamente en términos de aceleración, caracterizando el tamaño de los sismos por medio de la magnitud momento, $\mathrm{M}_{\mathrm{w}}$. El mapa de la Figura $2 \mathrm{~b}$ muestra el coeficiente de variación COV, que es adimensional y se define como el cociente de la desviación estándar dividido por la media de los resultados del árbol lógico, de modo que valores mayores de COV representan mayor variabilidad entre los resultados de las diferentes ramas, es decir, que son menos robustos. En Lorca la 
aceleración esperada es de $0.11 \mathrm{~g}$ (Figura 2a) pero el valor de COV es de 0.25 (Figura 2b), siendo éste valor el más alto de los encontrados en la región (variabilidad del $25 \%$ ). Es decir, el nivel de movimiento dado en Lorca lleva una elevada incertidumbre asociada, inherente especialmente a las diferentes zonificaciones que pueden adoptarse, y que generan importante variabilidad en los resultados.

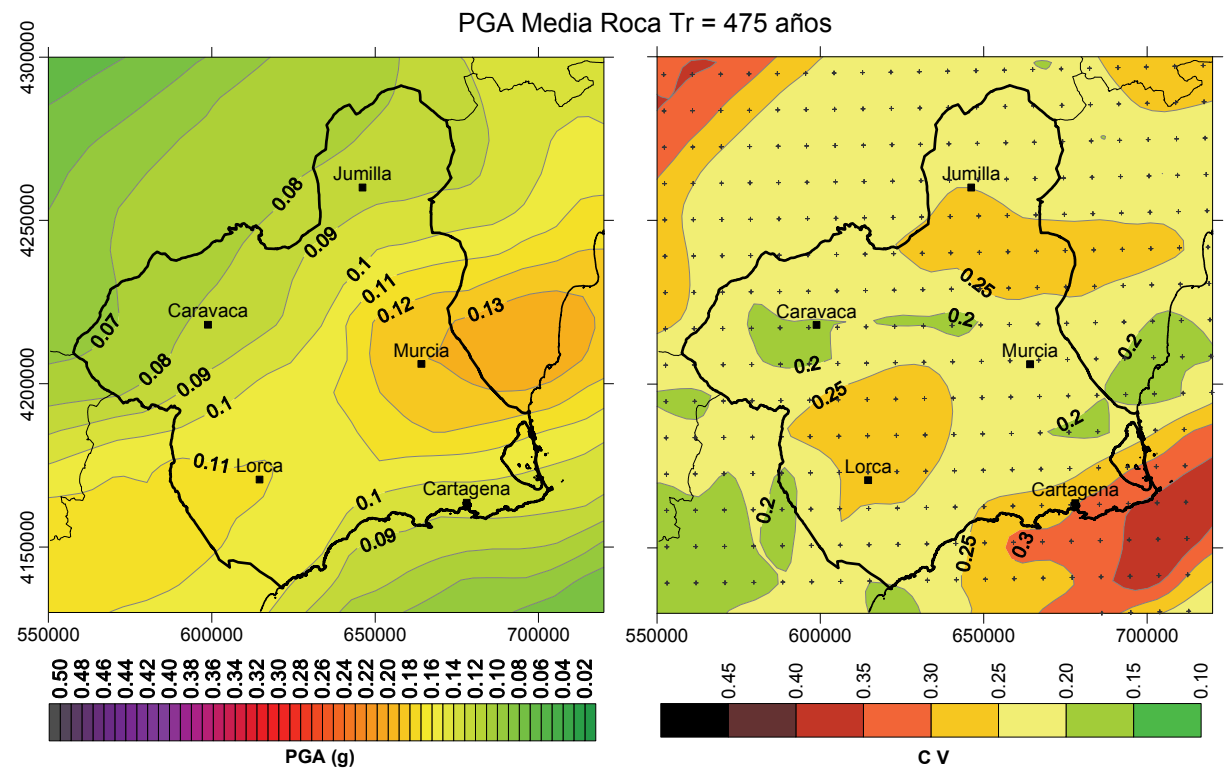

Fig. 2. Mapa de peligrosidad sísmica de la Región de Murcia en suelo tipo roca para periodo de retorno de 475 años (izq.) y mapa de coeficiente de variación asociado (dcha.). Fuente: RISMUR, Benito et al (2008).

En el mismo proyecto RISMUR se clasificaron los suelos de la provincia de Murcia y se asignaron factores de amplificación a cada suelo, que se superpusieron al mapa de aceleraciones en roca, dando como resultado el mapa de peligrosidad incluyendo el efecto local mostrado en la Figura 3.

El suelo en Lorca, a la escala regional del estudio, resulta ser tipo II según la clasificación de la NCSE-02 y la aceleración resultante al incluir el efecto local es de 0.13-0.16 g, que sigue estando por debajo de la PGA promedio en componente horizontal registrada en 2011. 


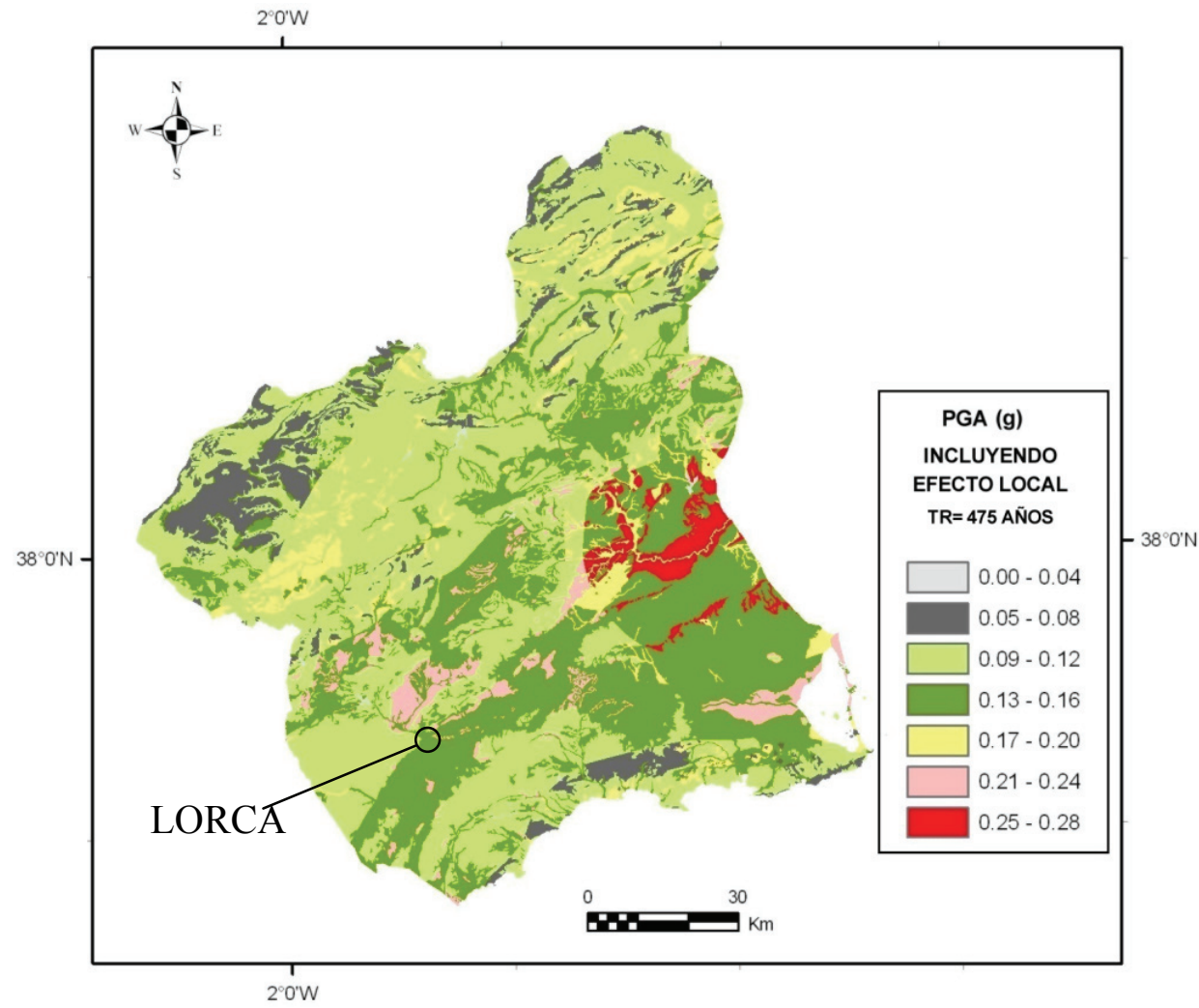

Fig. 3. Mapa de peligrosidad sísmica de la Región de Murcia incluyendo el efecto de sitio para periodo de retorno de 475 años. Fuente: RISMUR, Benito et al (2008).

No obstante, a la hora de situar el sismo de 2011 en el contexto de la peligrosidad sísmica, conviene tener en cuenta que los mapas probabilistas, como los de las Figuras 1 y 2, no corresponden a la aceleración dada por un terremoto particular, sino a la aceleración acumulada por eventuales sismos futuros en toda la región de influencia que puedan ocurrir en el tiempo asumido de 50 años. La aceleración esperada por un sismo específico se obtiene más bien desagregando la peligrosidad y deduciendo escenarios concretos asociados a los sismos de control que presenten mayor contribución.

Un estudio de este tipo fue también realizado en el proyecto RISMUR, resultando que para el movimiento objeto dado por la PGA obtenida previamente, el terremoto con mayor contribución en Lorca era un sismo moderado de $\mathrm{M}_{\mathrm{w}}$ entre 4.5 y 5.0 a corta distancia, entre 0 y $10 \mathrm{~km}$. El sismo de 2011 corresponde, precisamente, a este patrón de sismicidad, al igual que sucede con los sismos previos ocurridos en la provincia (en 1999, 2002 y 2005). Este importante resultado evidencia que, aunque en la zona cabe esperar la ocurrencia de terremotos más 
fuertes, hasta magnitud 6.5-7.0, son estos sismos moderados, próximos y frecuentes, los más determinantes, por presentar mayor contribución a periodos de retorno de 475 años, en el movimiento asumido para regulación de viviendas convencionales. De ahí que su estudio tenga especial relevancia en la revisión de la normativa. Para corroborar este resultado analizamos en el siguiente apartado la sismicidad histórica e instrumental de la provincia, lo que nos lleva a comprobar que sismos moderados y frecuentes constituyen un patrón de sismicidad típico en la región.

\subsection{Sismicidad histórica e instrumental en la provincia de Murcia}

La sismicidad histórica documentada en Murcia incluye varios terremotos dañinos en el pasado. La localización e intensidad de los sismos con I $\geq$ VI-VII EMS en la provincia, junto con los eventos ocurridos en época instrumental con $\mathrm{M}_{\mathrm{w}}$ mayor que 4.5, son mostrados en la Tabla 1, de acuerdo con Mezcua y Martínez Solares (1983), Martínez Solares y Mezcua (2002) y la página Web del IGN (www.ign.es). Los correspondientes epicentros se representan en el mapa de la Figura 4.

El primer terremoto reportado históricamente ocurrió en 1579, con intensidad VII MSK. Otro terremoto alcanzó Intensidad VIII EMS en 1674 y dos sismos más fueron reportados en 1911 con la misma intensidad, el 21 de marzo y el 3 de abril, causando importantes daños en Torres de Cotillas y Lorquí, respectivamente. Las magnitudes $\mathrm{M}_{\mathrm{S}}$ estimadas fueron de $5.7 \pm 0.5$ para el sismo de marzo y $5.3 \pm 0.3$ para el ocurrido en abril (Buforn et al, 2005).

Después de las series de 1911 no hay sismicidad significativa en la región de Murcia hasta el 23 de junio de 1948, cuando un terremoto de magnitud $\mathrm{M}_{\mathrm{w}} 5.3$ (Badal et al, 2000) tuvo su epicentro a distancia de $60 \mathrm{~km}$ al oeste de las series de 2011, según la localización dada por el IGN. La máxima intensidad asignada a este sismo fue de VIII Forel- Mercalli (Rey Pastor, 1949).

La actividad sísmica de la zona ha continuado durante el periodo instrumental, con varios terremotos de $M_{w} \geq 4.5$, desde 1972 (Tabla 1 y Figura 4). El periodo menos activo transcurrió entre 1948 y 1999, en el que tan solo se registraron 5 terremotos de magnitudes entre 4.0 y 4.5. Pero en Febrero de 1999 tuvo lugar un terremoto de $\mathrm{m}_{\mathrm{bLg}}=4.8$ con epicentro cerca de Mula y comenzó un periodo de mayor actividad en la región. Una intensidad máxima de VI (EMS) se alcanzó en Mula, Torres de Cotillas, Campos del Río y otras poblaciones a lo largo del río Mula (Buforn et al, 2005). Este terremoto fue objeto de varios estudios (IGN, 1999; Buforn and Sanz de Galdeano, 2001; Martínez-Díaz et al, 2002; Mancilla et al., 2002).

Otro sismo con $\mathrm{m}_{\mathrm{bLg}}=4.8$ (IGN) ocurrió en Agosto de 2002, cerca de Bullas, $20 \mathrm{~km}$ al oeste de Mula, alcanzando una Intensidad máxima de V (EMS) en Bullas y Cehegín y seguido por un gran número de réplicas, tres de ellas de $\mathrm{m}_{\mathrm{bLg}}$ $>3.5$. 
Tabla 1: Terremotos ocurridos en la Región de Murcia con magnitud $\mathrm{M}_{\mathrm{w}} \geq 4.5$ e intensidad $\mathrm{I} \geq \mathrm{VI}-\mathrm{VII}$.

\begin{tabular}{|c|c|c|c|c|c|c|}
\hline Fecha & Longitud & Latitud & Intensidad & $\mathbf{M}_{\mathbf{w}}$ & Origen $M_{w}$ & Localización \\
\hline $30 / 1 / 1579$ & -1.7000 & 37.6833 & VII & $5.4 *$ & IGN-I (EMS) & LORCA.MU \\
\hline $28 / 8 / 1674$ & -1.7000 & 37.6833 & VIII & $6.0 *$ & IGN-I (EMS) & LORCA.MU \\
\hline $9 / 3 / 1743$ & -1.1333 & 38.0000 & VII & $5.4 *$ & IGN-I (EMS) & MURCIA \\
\hline $15 / 8 / 1746$ & -1.1333 & 38.0000 & VI - VII & $5.1 *$ & IGN-I (EMS) & MURCIA \\
\hline $13 / 5 / 1756$ & -1.1333 & 37.9833 & VI - VII & $5.1 *$ & IGN-I (EMS) & MURCIA \\
\hline $20 / 12 / 1818$ & -1.6167 & 37.7500 & VI - VII & $5.1 *$ & IGN-I (EMS) & NE. LORCA.MU \\
\hline $11 / 11 / 1855$ & -1.4167 & 37.8667 & VI - VII & $5.1 *$ & IGN-I (EMS) & $\begin{array}{l}\text { ALHAMA DE } \\
\text { MURCIA.MU }\end{array}$ \\
\hline $16 / 4 / 1907$ & -1.5000 & 37.8000 & VII & $5.4 *$ & IGN-I (EMS) & TOTANA.MU \\
\hline $29 / 9 / 1908$ & -1.3000 & 38.1000 & VII & $5.4 *$ & IGN-I (EMS) & OJOS.MU \\
\hline $21 / 3 / 1911$ & -1.2167 & 38.0167 & VII - VIII & $5.7 *$ & IGN-I (EMS) & $\begin{array}{l}\text { LAS TORRES DE } \\
\text { COTILLAS.MU }\end{array}$ \\
\hline $3 / 4 / 1911$ & -1.2000 & 38.1000 & VII & $5.3 *$ & IGN-I (EMS) & LORQUI.MU \\
\hline $10 / 5 / 1911$ & -1.2000 & 38.1000 & VII & $5.4 *$ & IGN-I (EMS) & LORQUI.MU \\
\hline $16 / 5 / 1911$ & -1.2000 & 38.1000 & VII & $5.4 *$ & IGN-I (EMS) & LORQUI.MU \\
\hline $28 / 1 / 1917$ & -1.2667 & 38.0333 & VII & $5.4 *$ & IGN-I (EMS) & $\begin{array}{l}\text { LAS TORRES DE } \\
\text { COTILLAS.MU }\end{array}$ \\
\hline $13 / 6 / 1936$ & -1.4167 & 38.2333 & IV & $4.6 * *$ & $\begin{array}{l}\text { IGN-mD MMS } \\
(4.5)\end{array}$ & CIEZA.MU \\
\hline $23 / 6 / 1948$ & -1.7583 & 38.1417 & VIII & $5.2 * *$ & $\begin{array}{l}\text { IGN-mD MMS } \\
(5.2)\end{array}$ & CEHEGIN.MU \\
\hline $20 / 5 / 1952$ & -1.1000 & 37.9000 & V & $4.5 * *$ & $\begin{array}{l}\text { IGN-mD MMS } \\
(4.4)\end{array}$ & PALMAR.MU \\
\hline 2/2/1999 & -1.5014 & 38.0963 & VI & 4.8 & IAG-Mw & N MULA.MU \\
\hline $6 / 8 / 2002$ & -1.8353 & 37.8925 & $\mathrm{~V}$ & 5 & IGN-Mw & SW BULLAS.MU \\
\hline $29 / 1 / 2005$ & -1.7555 & 37.8535 & VII & 4.8 & IGN-Mw & NW ALEDO.MU \\
\hline $11 / 5 / 2011$ & -1.6756 & 37.6946 & VII & 5.1 & IGN-Mw & NE LORCA.MU \\
\hline $11 / 5 / 2011$ & -1.6812 & 37.7041 & VI & 4.5 & IGN-Mw & NE LORCA.MU \\
\hline
\end{tabular}

*Mw obtenida a partir del valor de intensidad, aplicando la correlación propuesta en Cabañas et al, 2012

**Mw obtenida a partir del valor de $m_{b L g}$, (dado por Mezcua y Martínez-Solares 1983 a partir del dato de magnitud duración $m_{D}$ ) aplicando la correlación propuesta por Cabañas et al, 2012.

El último terremoto dañino en la zona, previo al de Lorca 2011, ocurrió el 29 de enero de 2005, con epicentro localizado cerca de la población de Avilés y $\mathrm{m}_{\mathrm{bLg}}$ 4.7. Este sismo causó importantes daños en La Paca y Zarcilla de Ramos, alcanzando una Intensidad VII (EMS-98) y desencadenando pérdidas económicas por 
encima de 10 millones de euros (Benito et al, 2007). Hay evidencias de que este sismo pudo ser disparado por el de Bullas en 2002.

Por tanto, el análisis de la sismicidad histórica e instrumental confirma un patrón de sismicidad observado en la región compuesto por sismos que tienen lugar, con relativa frecuencia, con magnitudes entorno a $\mathrm{M}_{\mathrm{w}} 5.0$ y que causan importantes daños, llegando a Intensidades máximas de I=VIII (EMS). Ocasionalmente se han observado agrupaciones temporales de esos sismos, ocurriendo en plazos de 1 a 3 años entre dos sismos consecutivos, y alternándose estas agrupaciones con otros periodos mayores de calma.

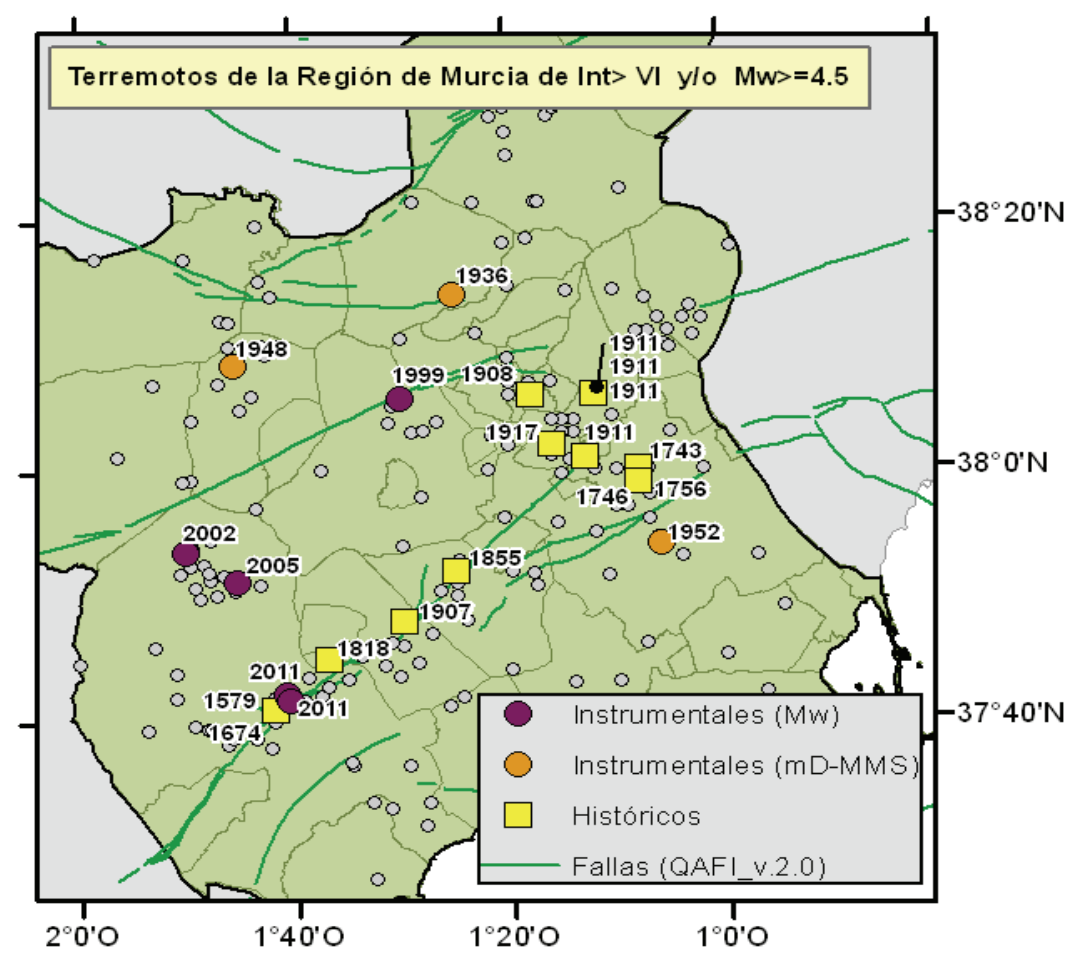

Fig. 4. Terremotos ocurridos en la Región de Murcia con magnitud con magnitud $M_{w} \geq 4.5$ e intensidad I $\geq$ VI-VII, junto con las fallas activas (QAFI v. 2.0).

\subsection{Movimiento fuerte registrado en Lorca 2011 y predicho en análogas condiciones}

El sismo de Lorca 2011 ha generado un importante número de acelerogramas, que arrojan abundante información para analizar la atenuación en la zona y, más concretamente, para calibrar las denominadas Ecuaciones de Predicción del 
Movimiento Fuerte (GMPE's). Resulta particularmente interesante, de cara a futuras estimaciones de peligrosidad, conocer cómo se distribuyen los valores de PGA y $\mathrm{SA}(\mathrm{T})$ registrados a diferentes distancias respecto a los simulados con diferentes GMPE's, y extraer conclusiones al respecto.

Con ese objetivo comparamos, en primer lugar, los datos de PGA registrados con los predichos por varias GMPE's seleccionadas, para el rango de distancias en el que existen registros. En segundo lugar, efectuamos una comparación de los espectros de respuesta derivados de los registros en la estación de Lorca, a $3 \mathrm{~km}$ de distancia epicentral, con los propuestos por la NCSE-02 para esta población y con los deducidos en el proyecto RISMUR.

La Tabla 2 muestra los valores registrados de PGA para el sismo principal en las componentes horizontales, en 11 estaciones a distancias comprendidas entre 3 y $185 \mathrm{~km}$. Los máximos valores de PGA en Lorca fueron de 0.365 y $0.153 \mathrm{~g}$ para las componentes horizontales N30W y E30N, respectivamente. Esos valores son los mayores registrados en la Península Ibérica, como se deduce analizando las bases de datos del IGN. Esto puede ser explicado como consecuencia de la proximidad y somera profundidad de la superficie de ruptura, probablemente con algún efecto añadido de directividad que da lugar a un movimiento impulsivo de corta duración (ver López Comino et al. 2012, Martínez-Díaz et al. 2012).

Tabla 2. Valores pico de aceleración (PGA) del terremoto principal de la serie (11 de mayo de 2011) registrados en estaciones del IGN.

\begin{tabular}{|l|r|r|r|}
\cline { 2 - 4 } \multicolumn{1}{c|}{} & \multicolumn{3}{c|}{ PGA horizontal (g) } \\
\hline Estaciones & Comp. Norte & Comp. Este & \multicolumn{1}{c|}{ Media geométrica } \\
\hline Lorca & 0.365 & 0.153 & 0.236 \\
\hline Alhama de Murcia 01 & 0.042 & 0.045 & 0.043 \\
\hline Alhama de Murcia 02 & 0.010 & 0.008 & 0.009 \\
\hline Zarcilla de Ramos & 0.026 & 0.032 & 0.029 \\
\hline Mula & 0.036 & 0.042 & 0.039 \\
\hline Vélez-Rubio & 0.011 & 0.009 & 0.010 \\
\hline Lorquí & 0.008 & 0.008 & 0.008 \\
\hline Murcia & 0.007 & 0.009 & 0.008 \\
\hline Fortuna & 0.007 & 0.008 & 0.007 \\
\hline Jumilla & 0.004 & 0.005 & 0.004 \\
\hline Cieza & 0.002 & 0.003 & 0.003 \\
\hline
\end{tabular}

En la Figura 5 se muestran los registros acelerométricos del terremoto principal en distintas estaciones del IGN, pudiendo apreciarse la variabilidad en las amplitudes y la atenuación con la distancia. 
En general, la componente denominada N30W es la que registra las amplitudes mayores, tanto en el sismo principal como en el premonitor y la réplica. En particular, en el terremoto principal, esta componente presenta una fase de movimiento fuerte muy acusada, con una sucesión de picos por encima de $0.2 \mathrm{~g}$ en menos de $2 \mathrm{~s}$ de duración.

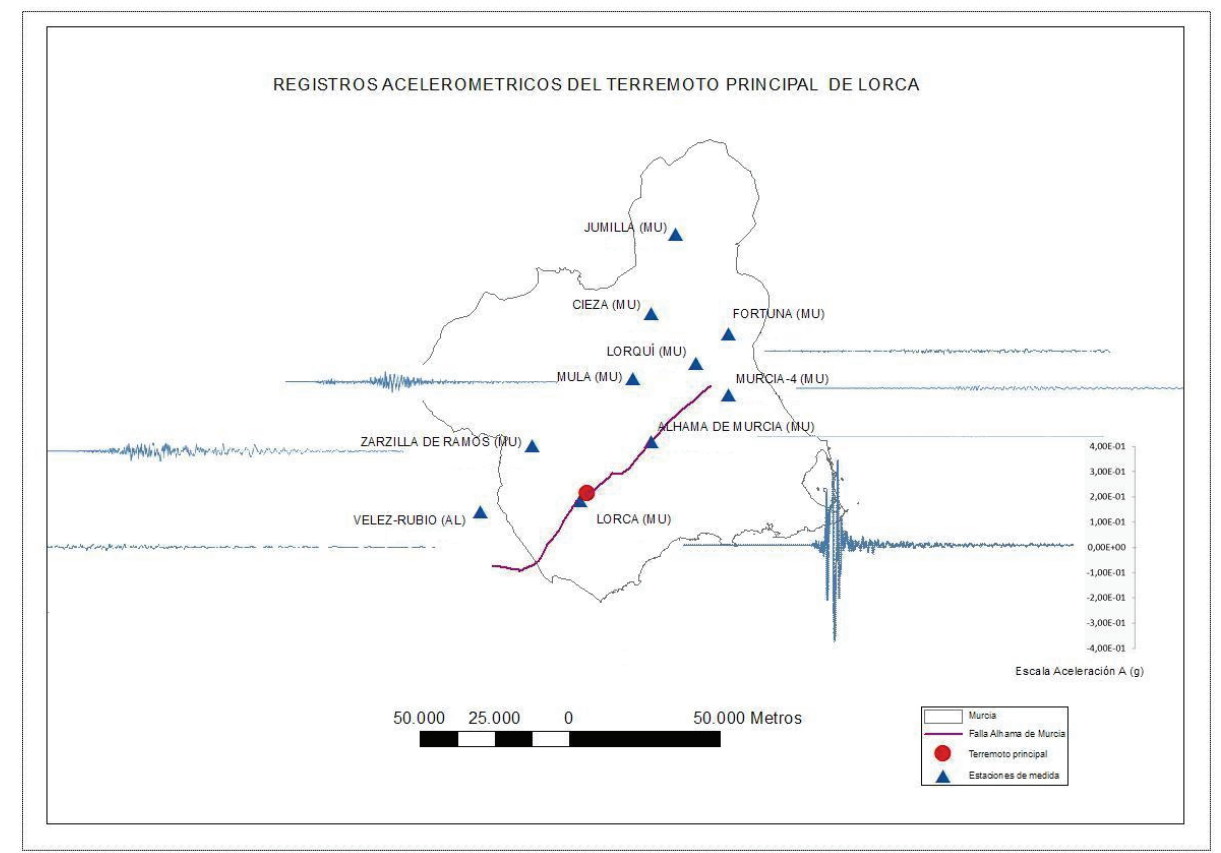

Fig. 5. Distribución geográfica de registros de movimiento fuerte en estaciones del IGN para el evento principal del 11 de mayo de 2011 con $\mathrm{M}_{\mathrm{w}}$ 5.1. Se representa la componente horizontal máxima en cada estación.

Otro factor importante a tener en cuenta en la amplitud registrada, es el efecto de las condiciones locales del suelo en el emplazamiento de la correspondiente estación acelerométrica. Este parece ser el caso (aunque requiere un estudio más detallado) de los registros de las dos estaciones de Alhama de Murcia (ver Tabla 2). Ambas estaciones, prácticamente a distancias similares del epicentro, están situadas en emplazamientos de características bien diferentes. El acelerómetro situado más próximo al centro de la cuenca del Guadalentín, es el que ha registrado una mayor amplitud del movimiento, posiblemente como consecuencia de la amplificación del terreno aluvial con mayor potencia, en el que se encuentra. En todo caso será necesaria una caracterización completa del suelo de estos emplazamientos. 
Tal y como se ha visto, el registro del terremoto principal en Lorca alcanza aceleraciones muy superiores a las registradas en el resto de estaciones. Este registro está situado sobre el plano de ruptura, a una distancia $\mathrm{R}_{\mathrm{JB}}$ nula (distancia Joyner y Boore o distancia más corta a la proyección en superficie del plano de ruptura).

Con objeto de valorar si los niveles de movimiento registrado se ajustan o no a los predichos habitualmente de forma empírica, por medio de los modelos de movimiento fuerte que se utilizan después en el cálculo de la peligrosidad, se presenta una simulación del movimiento esperado en la ciudad de Lorca. Esta simulación se realiza bajo la hipótesis de que el plano de ruptura del evento principal del 11 de mayo de 2011, está situado en la falla de Alhama de Murcia, coincidiendo con uno de los planos nodales de la solución del mecanismo focal obtenida por el IGN (Figura 6). Además, para la predicción del movimiento se adoptan los modelos existentes en la literatura (GMPE's), que reúnen más consenso científico y al mismo tiempo resultan más adecuados para la zona en la que se realiza la aplicación.

Las características del plano de ruptura son:

- Geometría Rectangular

- Techo ruptura a una profundidad de $1 \mathrm{~km}$

- Longitud (L) $3.451 \mathrm{~km}$

- Anchura (W) $3.027 \mathrm{~km}$

- Azimut 230

- $\quad$ Buzamiento (dip) $69^{\circ}$

- Deslizamiento (rake) $33^{\circ}$

- Coordenadas epicentro (UTM) X=617029 m Y=4173277 m

- $\quad$ Magnitud $\mathrm{M}_{\mathrm{w}}=5.1$

\subsubsection{Simulación de aceleraciones pico, PGA}

Se utilizan varias GMPE's para estimar la atenuación de la PGA con la distancia, en concreto tres modelos NGA (Abrahamson y Silva 2008, Chiou y Youngs 2008 y Campbell y Bozorgnia 2008) - elegidos por el grado de detalle que permiten introducir en la modelización de la fuente- $\mathrm{y}$ un modelo europeo reciente (Akkar y Bommer 2010). Se ha considerado un tipo de suelo uniforme con velocidad de onda de cizalla promedio para los 30 primeros metros $\mathrm{v}_{\mathrm{s} 30}$ de $1100 \mathrm{~m} / \mathrm{s}$, lo que equivale a considerar un suelo tipo roca. Los resultados se muestran en la Figura 7, donde puede observarse la aceleración predicha por cada modelo y la distribución de estaciones de registro, indicando aquellas estaciones cuyos registros son superados por los valores del modelo (en verde) y aquellas en las que sucede lo contrario (en rojo). Los modelos de Abrahamson y Silva 2008 y Chiou y Youngs 2008 son los que presentan una atenuación más rápida de la PGA con la distancia. 


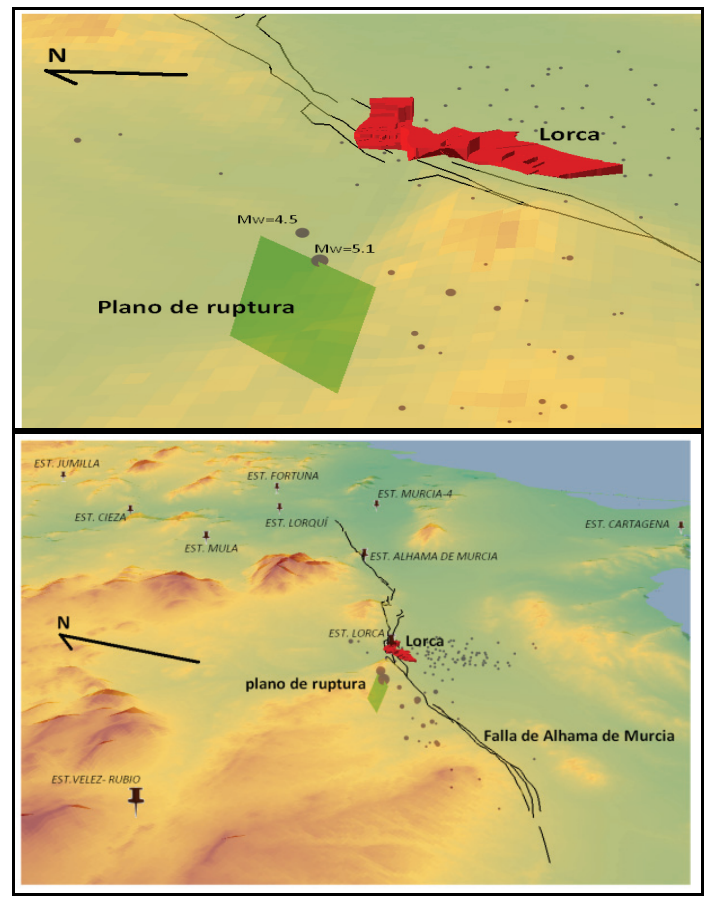

Fig. 6. Vistas del plano de ruptura del sismo principal del 11 de mayo de 2011 (verde) y localización del núcleo urbano de Lorca (rojo).

En la Figura 8 se representa una sección en dirección aproximada N30E (dirección de la componente horizontal máxima), en la que se representan las diversas curvas de atenuación y los datos registrados en las diferentes estaciones, junto con los valores de las dos componentes geométricas horizontales y de la media geométrica de ambas. Se observa que los modelos de Chiou y Youngs 2008 y de Campbell y Bozorgnia (2008) son los que dan valores de aceleración más altos a distancias muy cortas. En el caso de las estaciones de Lorca y de Mula, los modelos no pueden predecir el valor de PGA horizontal máximo registrado en componentes individuales (NS), de modo que los valores registrados exceden (en general notablemente) a los valores predichos. Para distancia superiores a $20 \mathrm{~km}$, lejos del plano de ruptura, las aceleraciones registradas son del mismo orden o incluso inferiores a las predichas por los modelos, como norma general. 


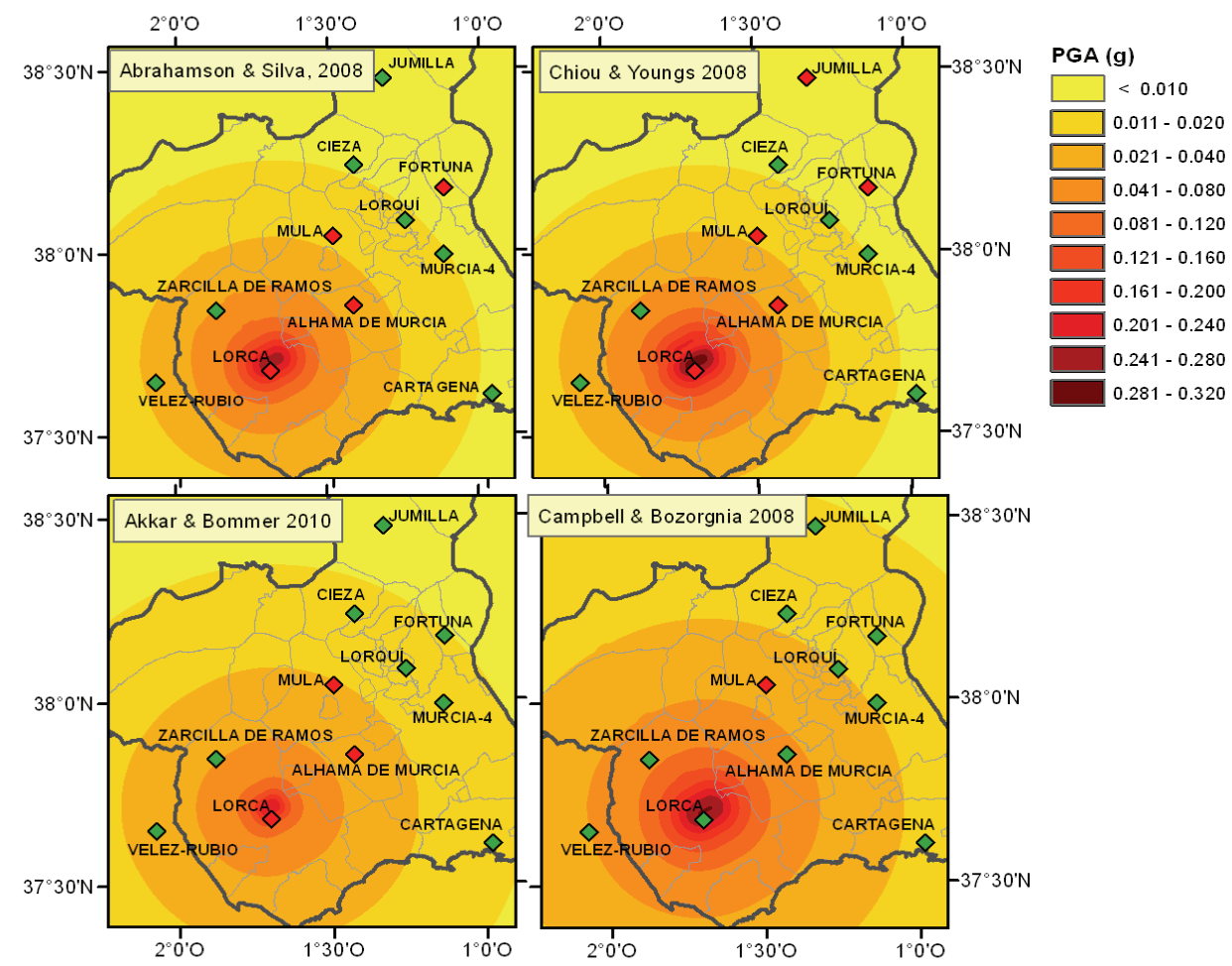

Fig. 7. PGA estimada para el sismo principal del 11 de mayo de 2011 con los modelos de Abrahamson y Silva 2008, Akkar y Bommer 2010, Campbell y Bozorgnia 2008 y Chiou y Youngs 2008) junto a las estaciones de registro. Se representan en color verde las estaciones que registran valores inferiores al dado por el modelo y en rojo aquellas que registran valores superiores a éste.

La Figura 8 evidencia lo ya comentado: en general, los modelos sobrestiman el movimiento para distancias superiores a $10 \mathrm{~km}$, si bien en algunos casos resultan predicciones muy satisfactorias, como sucede con alguna de las GMPE's considerada y los valores registrados en las estaciones de Alhama de Murcia, Lorquí, Fortuna y Jumilla. A corta distancia, $\mathrm{r}_{\mathrm{epi}} 3 \mathrm{~km}$ y $\mathrm{r}_{\mathrm{JB}}$ nula (es decir, sobre el plano de ruptura), el valor registrado en la componente NS excede notablemente los valores predichos por todos los modelos, si bien la componente EW queda por debajo de todos ellos. En este caso, los modelos predicen razonablemente bien la media geométrica de las dos componentes horizontales. 


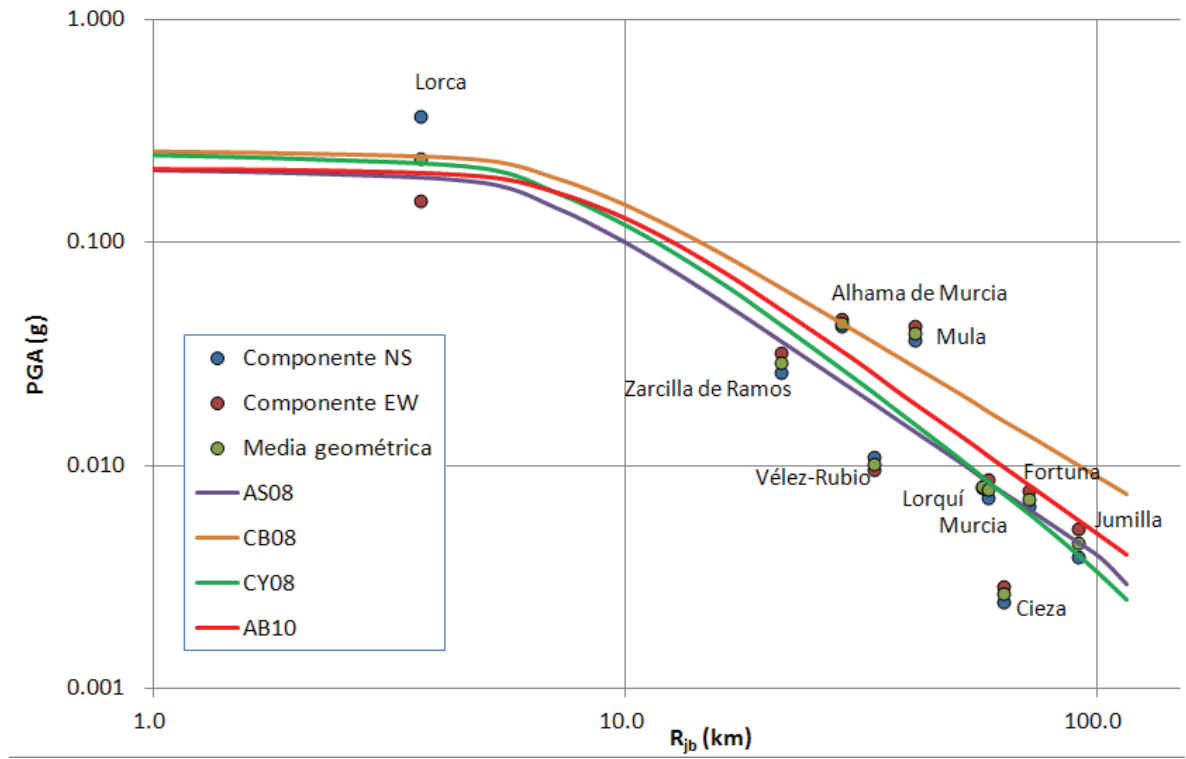

Fig. 8. Valores de aceleración pico (PGA) registrados en diversas estaciones para el terremoto de Lorca de 2011 (símbolos puntuales), junto con los valores predichos por los modelos de movimiento fuerte considerados en análogas condiciones de magnitud y distancia para emplazamiento tipo roca.

Un hecho similar ha sido observado en otros terremotos recientes, con magnitud moderada como L'Aquila (2009) de Mw 6.3 y Christchurch (2011) también de $\mathrm{Mw}$ 6.3. Las aceleraciones registradas dentro del plano de falla se disparan, siendo muy superiores a las predichas por los modelos, y sin embargo decrecen con rápida atenuación a distancias superiores a $20 \mathrm{~km}$. Todo parece confirmar la existencia de un cierto "Efecto fuente", que puede ser debido a directividad de la ruptura, fases de parada $u$ otros factores a investigar, dando como resultado aceleraciones anormalmente altas dentro del plano de falla, al menos en una componente del movimiento. Estas aceleraciones no son bien predichas por los modelos de movimiento fuerte (que normalmente predicen la media geométrica de las componentes horizontales), generalmente deducidos por ajuste de regresión de datos reales a distancias mayores, de modo que el rango de distancias cortas correspondientes al plano de ruptura- no queda bien cubierto por los modelos. También puede deberse a un efecto de la profundidad, que se manifiesta en sismos muy superficiales. Algunos de los modelos (Akkar y Bommer 2010) se desarrollan para profundidades medias, del orden de $8 \mathrm{~km}$, y si el sismo tiene profundidad muy inferior (como el caso de Lorca donde se estima que ésta fue de $1 \mathrm{a} 3 \mathrm{~km}$ ) esa diferencia puede explicar también la diferencia en las aceleraciones 
predicha y registrada. En el campo próximo la aceleración es muy sensible a la profundidad del sismo.

Este hecho tiene una gran trascendencia en el cálculo de la peligrosidad, basado en la estimación de la aceleración por medio de modelos de movimiento fuerte. Si esos modelos infraestiman la aceleración en el plano de ruptura, la peligrosidad que resulta en el entorno de la falla es también infraestimada y las correspondientes aceleraciones son menores que las reales. El caso tiene una importancia especial cuando una población se encuentra justo en el plano de la falla, como ocurre en Lorca, porque entonces las aceleraciones que se introducen en el diseño pueden estar siendo muy inferiores a las que genera el terremoto a distancias muy cortas. Este podría ser un factor que contribuye a explicar el importante daño observado en estructuras recientes, construidas supuestamente al amparo de la normativa.

\subsubsection{Simulación de espectros de respuesta}

La Figura 9 muestra los espectros de respuesta derivados de los registros de la componente horizontal máxima, tanto para el sismo principal como para el premonitor, junto con el dado en Lorca por la NCSE-02 y los deducidos en el proyecto RISMUR para suelo duro y para roca. El análisis de los espectros de respuesta registrados, señala un máximo en la banda de periodos de vibración entre 0.2 y aproximadamente 0.5 segundos, con amplitudes en el rango de 0.5 a $\quad 0.65$ $\mathrm{g}$ para las correspondientes aceleraciones espectrales SA(T). Estas amplitudes son notablemente mayores en el caso del terremoto principal que en el caso del premonitor. En el rango de periodos indicado, los espectros registrados en la estación de Lorca superan claramente (en un factor 2 o superior) a los espectros de la norma NCSE-02 y a los espectros derivados del proyecto RISMUR para la ciudad de Lorca.

Asimismo se han comparado los espectros de respuesta calculados usando varias GMPE's ecuaciones con el espectro de la media geométrica de las componentes horizontales, para el caso del registro del evento principal en la estación de Lorca (Figura 10). Se observa que los espectros calculados con los modelos superan al del registro solamente en el rango de bajos periodos (hasta $0.2 \mathrm{~s}$ ). Para periodos superiores, el espectro registrado es claramente superior al espectro medio predicho por los modelos, con un pico en torno a 0.4-0.5 s. De hecho en este intervalo espectral el espectro registrado se acerca más al espectro medio más una desviación estándar de dados por los modelos. 


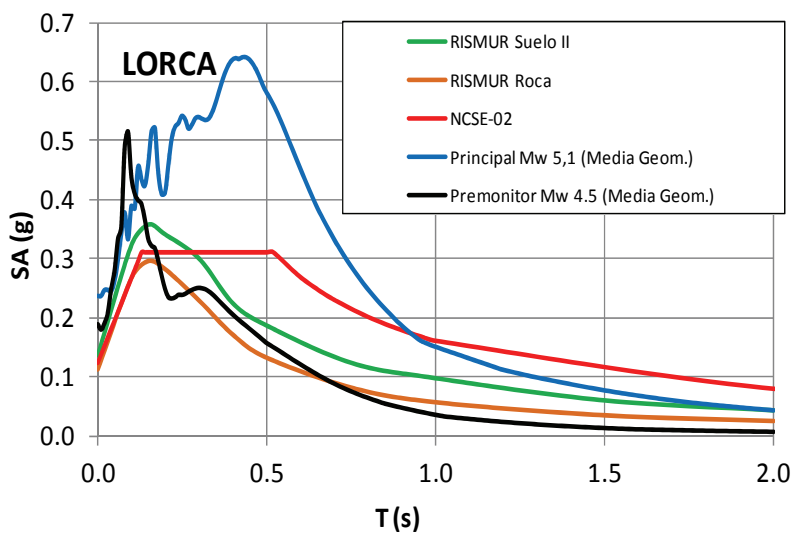

Fig. 9. Espectro de respuesta registrado en la estación de Lorca para el premonitor y para el sismo principal (media geométrica), junto con el espectro de la Norma NCSE-02 y los espectros obtenidos en RISMUR para emplazamiento tipo I (roca) y II (suelo duro), según la clasificación de la NCSE-02.
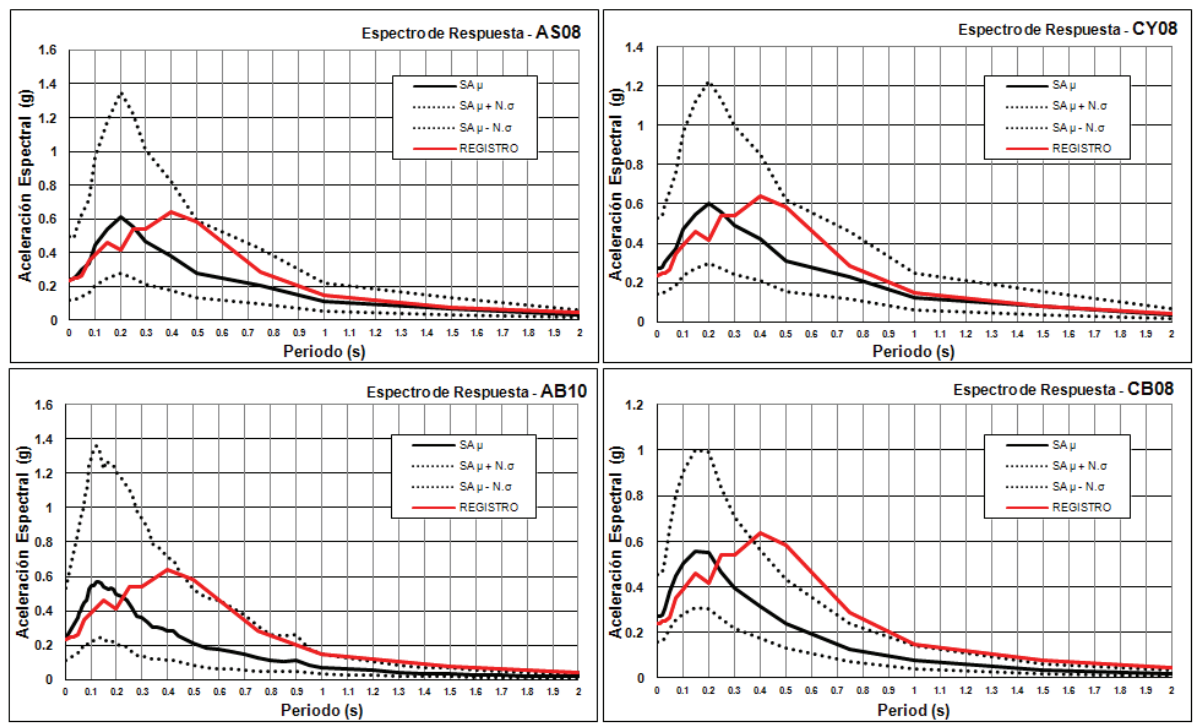

Fig. 10. Espectro de respuesta registrados en la estación de Lorca (media geométrica de las dos componentes horizontales del sismo principal, en rojo) y espectros promedio ( \pm 1 sigma) calculado con los mismos GMPE de la Figura 7. 


\subsubsection{Desagregación: sismos de control}

Los terremotos de control, que dominan la peligrosidad para la probabilidad de excedencia asumida (10\% en 50 años), corresponden a eventos de magnitud bajamoderada $\mathrm{M}_{\mathrm{w}}(4.5-5.0)$ con distancias fuente-emplazamiento cortas $(0-10 \mathrm{~km})$ (Gaspar-Escribano et al. 2008), es decir, similares al sismo principal y al premonitor del 11 de mayo de 2011 en Lorca. De este modo se constata que el patrón de sismicidad observado en la zona, compuesto por sismos frecuentes de magnitud en torno a 5.0, constituye la mayor contribución a la peligrosidad para la probabilidad que se considera oficialmente en el diseño sismorresistente de viviendas convencionales.

Asimismo, diferentes espectros de respuesta estimados en estudios recientes, incluidos los asociados a los citados sismos de control, superan a los correspondientes espectros de la norma NCSE-02 en el rango de los cortos periodos, siendo sin embargo superados por ésta para los periodos largos (Gaspar-Escribano y Benito, 2007). Todos están muy por debajo del registrado en la estación de Lorca en el sismo principal del 11 de mayo de 2011, lo que podría contribuir a explicar el daño causado especialmente en viviendas de entre 3 y 5 plantas, con periodo fundamental de vibración en el rango de los cortos periodos.

\subsection{Conclusiones sobre el movimiento registrado en el marco de la peligrosidad}

Resumiendo las principales evidencias puestas de manifiesto en relación con el movimiento fuerte, cabe destacar:

- El movimiento registrado en la estación de Lorca, por el sismo principal del 11 de mayo, con un valor de PGA de $0.37 \mathrm{~g}$ para la componente horizontal N30W excedió el dado por la NCSE-02 y por los derivados de diferentes estudios de peligrosidad en la región, para 475 años de periodo de retorno, por un factor 3; si bien el registrado en la componente E30N fue notablemente menor (en torno a $0.15 \mathrm{~g}$ ) lo que hace que, si se considera el promedio de las dos componentes horizontales, ese factor de excedencia se reduzca a 2 .

- La elevada aceleración registrada en componente N30W está también por encima de la que predicen los modelos de movimiento fuerte en el plano de ruptura, lo que se explica por un cierto efecto fuente, debido seguramente a directividad, no capturado por los modelos de predicción. En el caso de Lorca 2011, la somera profundidad del foco a tan solo $3 \mathrm{~km}$, puede contribuir también a explicar la excedencia del movimiento registrado sobre el predicho, dado que la mayor parte de la GMPE's consideran profundidades medias de $10 \mathrm{~km}$.

- En cualquier caso, la alta aceleración registrada en una componente, superando la resultante de cualquier estudio de peligrosidad con cualquier modelo de movimiento, lleva a plantear dos cuestiones fundamentales:

1.Si es acertado emplear GMPE's en peligrosidad basados en estimación del promedio de las dos componentes horizontales, o deberían usarse 
modelos de predicción de la componente máxima, en un supuesto más conservador.

2.Si la aceleración registrada en Lorca corresponde al 10\% de probabilidad de excedencia en 50 años que se asume cuando se adoptan periodos de retorno de 475 años. A este respecto se podrían considerar varios factores: la profundidad del foco, anormalmente baja y el carácter tan impulsivo del movimiento registrado, con elevadas aceleraciones y muy corta duración. Cabe preguntarse si ese es un patrón de ruptura típico en ese segmento de la falla de Alhama de Murcia, o puede calificarse de anómalo, tanto por la profundidad como por el patrón de radiación.

- Por otra parte, el terremoto de $\mathrm{M}_{\mathrm{w}} 5.1$ y su premonitor, de $\mathrm{M}_{\mathrm{w}} 4.5$, son coherentes con los sismos de control que han resultado en análisis previos de peligrosidad para 475 años de periodo de retorno: sismos de magnitud moderada, $\mathrm{M}_{\mathrm{w}}(4.5$ - 5.0), a cortas distancias, $(0 \mathrm{y} 10 \mathrm{~km})$. Los tres sismos previos de Mula, 1999; Bullas, 2002 y La Paca, 2005, vienen a corroborar este patrón de sismicidad, al tiempo que refuerzan la hipótesis de posibles mecanismos de disparo entre eventos en la zona. La sismicidad muestra agrupaciones -como la de los cuatro últimos sismos en tan solo 12 años-, que se alternan con periodos de calma - como el trascurrido entre 1948 y 1999-, periodo en el que únicamente se registraron cinco eventos con magnitudes $\mathrm{m}_{\mathrm{bLg}}$ entre 4.0 y 4.5 .

- Por último, los espectros de respuesta registrados en Lorca para el sismo principal presentan máximos en la banda de periodos de vibración entre 0.2 y 0.5 segundos, con aceleraciones espectrales en el rango de 0.5 a $0.65 \mathrm{~g}$. Estos superan claramente a los espectros de la norma NCSE-02 y a los derivados del proyecto RISMUR para la ciudad de Lorca, así como a los predichos por diferentes GMPE's para un escenario similar. Nuevamente cabe preguntarse si el espectro registrado corresponde (o no) al 10\% de probabilidad de excedencia en 50 años que suponen los demás que se están comparando.

\section{Riesgo sísmico en Lorca y daño observado}

\subsection{El riesgo de Lorca enmarcado en el riesgo de la provincia de Murcia}

El proyecto RISMUR proporciona una estimación del daño acumulado esperado en todos los municipios de Murcia para un periodo de retorno de 475 años. Si bien este daño acumulado no se puede asociar al que produciría un solo terremoto, sí puede ser utilizado para comparar el daño relativo entre diferentes localidades expuestas a un mismo régimen de sismicidad. En la Tabla 3 se muestra la distribución de daño obtenida para el municipio de Lorca y en ella se diferencian tres grados de daño (leve, moderado y grave) en las edificaciones del municipio. De acuerdo con los resultados del proyecto RISMUR, Lorca es una de las poblaciones que sufrirían mayor daño medio esperado de la región y la octava con mayor daño neto, representado por el número de edificios que quedarán inhabitables por 
el movimiento sísmico esperado (con $10 \%$ de probabilidad de excedencia en 50 años), resultando un total 663 edificios (Figuras 11 y 12).
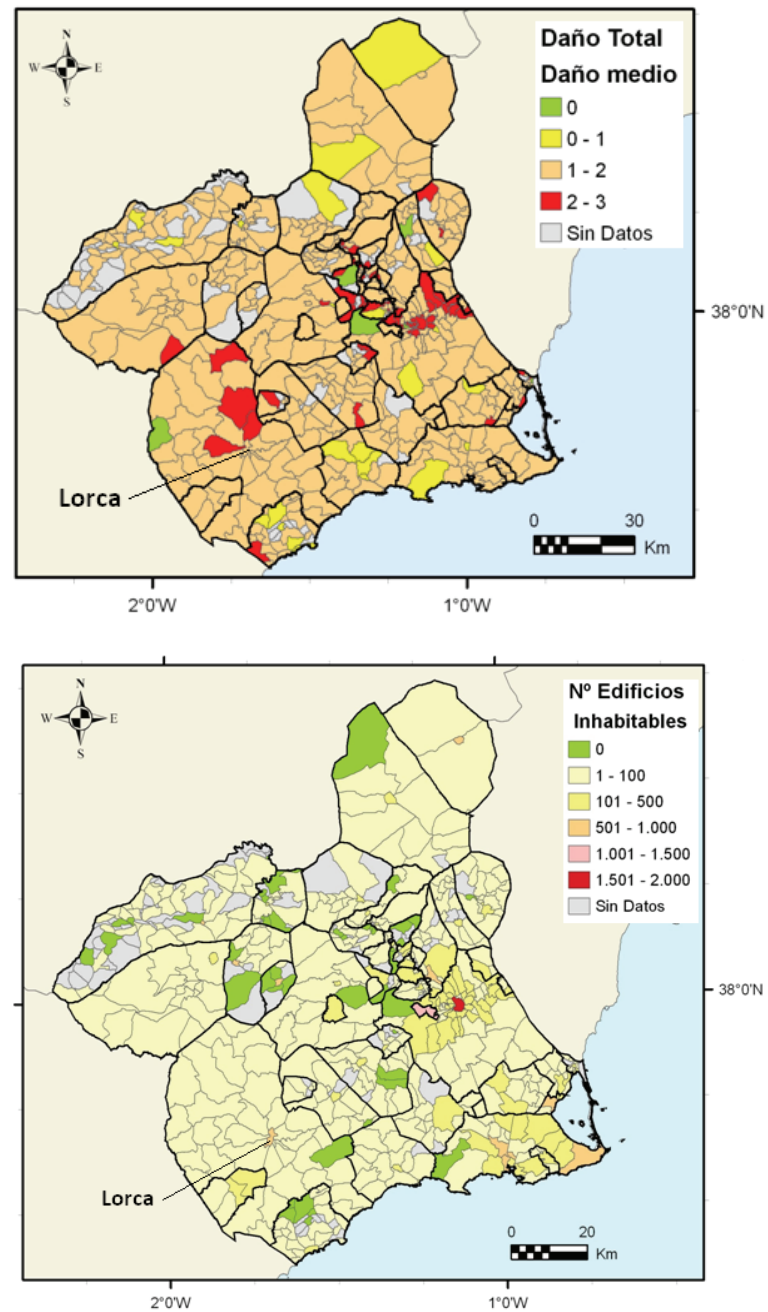

Fig. 11. Daño estimado en la Región de Murcia para un periodo de retorno de 475 años, según los resultados del estudio RISMUR y Benito et al. (2008). Arriba: daño medio; abajo número de edificios inhabitables para el movimiento esperado con $10 \%$ de probabilidad de excedencia en 50 años.

Para contextualizar el riesgo de Lorca dentro de la provincia de Murcia, la figura 12 muestra los 22 municipios que resultan con mayor daño neto, en función 
del número de inhabitables y la Figura 13 muestra la distribución de daño esperado grave, moderado y leve en las poblaciones que resultan con mayor número de edificios con daño grave. Lorca se encuentra en $8^{\mathrm{a}}$ y $9^{\mathrm{a}}$ posición, respectivamente, en estos baremos, dentro del conjunto de municipios de la provincia. Los primeros lugares de ambos lo ocupan Murcia y Alcantarilla, si bien hay que tener en cuenta que, al tratarse de daño neto, el número total de edificios es determinante.

Tabla 3. Número de edificios con distinto grado de daño esperado en Lorca según resultados de RISMUR para un periodo de retorno de 475 años. D0 - Daño nulo, D1 - Daño leve, D2 - Daño moderado, D3 - Daño sustancial a fuerte, D4 - Daño muy fuerte, D5 - Destrucción (según los grados de daño diferenciados en la escala EMS-98).

\begin{tabular}{|c|c|c|}
\hline Tipo de daño & Número de edificios & \% de edificios \\
\hline Leve (D0+D1) & 3592 & $56 \%$ \\
\hline Moderado (D2+D3) & 2583 & $40 \%$ \\
\hline Grave (D4+D5) & 247 & $4 \%$ \\
\hline
\end{tabular}

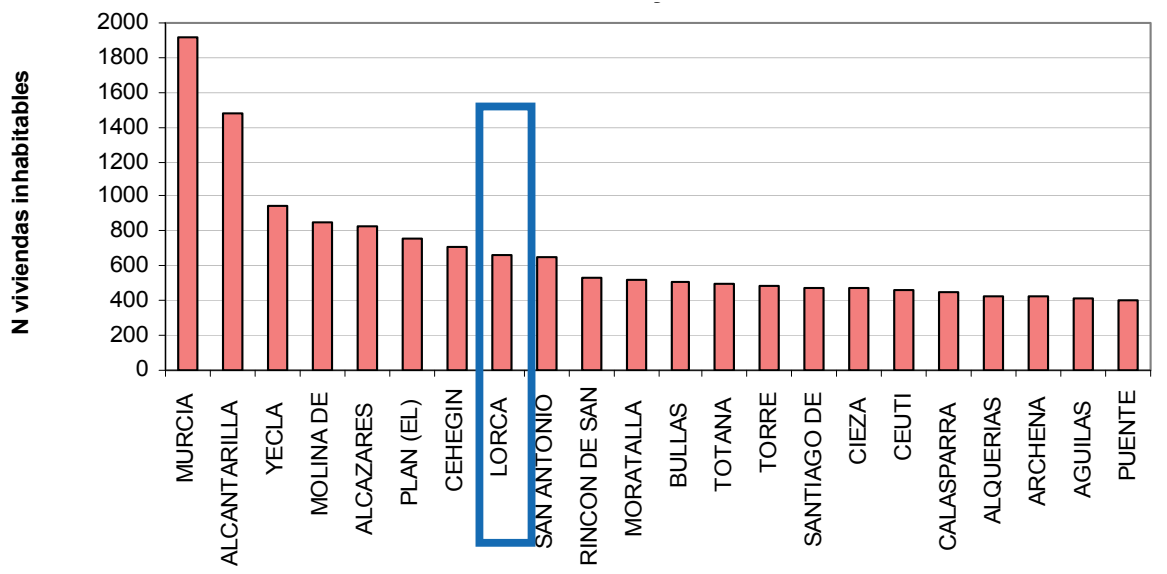

Fig. 12. Número esperado de edificios inhabitables en los 22 municipios de Murcia que resultan con mayor daño neto, para un periodo de retorno de 475 años (estudio RISMUR y Benito et al. 2008). 


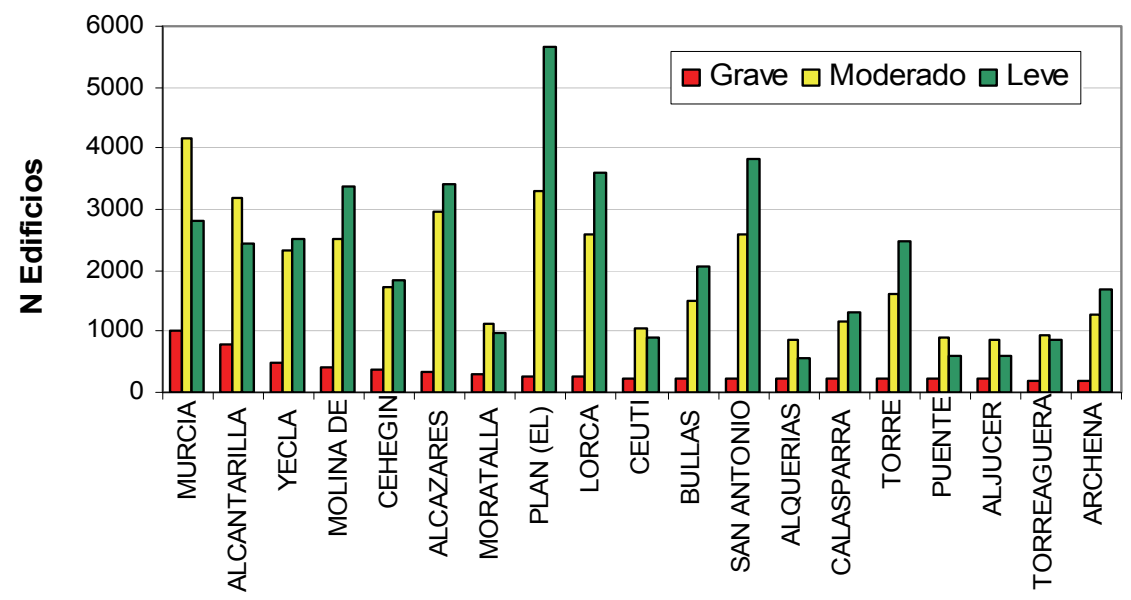

Población

Fig. 13. Representación de Daño Leve, Moderado y Grave en las poblaciones con mayor daño grave. Fuente: RISMUR.

\subsection{Lorca: población con prioridad de actuación según los resultados del Proyecto RISMUR.}

Las Figuras 12 y 13 muestran un resultado en parte esperado: los municipios con mayor daño neto corresponden a aquellos con mayor número de edificios construidos. La pregunta que cabe formularse es: ¿Cuáles de los municipios con mayor daño esperado deberían priorizarse para el desarrollo de estudios de detalle a escala municipal hacia la mitigación del riesgo sísmico? Para responder a esta cuestión, en el proyecto RISMUR se introdujo un criterio combinado en el que, además del daño esperado, se tuvieran en cuenta otros factores a la hora de establecer esa prioridad. Se consideraron así dos elementos de ponderación nuevos: la cercanía a una falla activa y el estado de esfuerzos en la corteza, deducido a partir de la transferencia de esfuerzos estáticos de Coulomb a causa de los terremotos ocurridos en el pasado. Así, en el mapa de la Figura 14 se observa que existen varios segmentos de fallas activas de dimensiones considerables (líneas rojas) que han sido cargados de esfuerzos de Coulomb y que favorecen la reactivación de los mismos (zonas rojas). Entre estos sectores destacan los segmentos de Campo Coy y Fortuna de la Falla de Crevillente; el sector de Calasparra de la falla SocovosCalasparra y El sector de Alcantarilla de la Falla de Alhama de Murcia; el segmento Puerto Lumbreras-Lorca de la Falla de Alhama de Murcia y el sector Norte de la Falla de Palomares.

De acuerdo con estos parámetros, en el Proyecto RISMUR se planteó el siguiente esquema de prioridades, partiendo siempre de las poblaciones con mayor daño neto (identificadas en Figuras 12 y 13): 
- Prioridad 1: ubicadas en zonas de incremento de esfuerzos y próximas a alguna falla principal.

- Prioridad 2: ubicadas en zonas de incremento de esfuerzos pero distantes de alguna falla principal.

- Prioridad 3: ubicadas fuera de zonas de incremento de esfuerzos pero próximas a alguna falla principal.

- Prioridad 4: ubicadas fuera de zonas de incremento de esfuerzos y que no estén próximas a alguna falla principal.

En la Figura 14, aparecen junto a los lóbulos de máximo incremento de esfuerzos y las grandes fallas de la región, las poblaciones con mayor daño esperado (estrellas amarillas). La inspección del mapa permite visualizar la situación de dichas poblaciones, relativa a las principales fallas activas y a su vez a las zonas con mayor probabilidad de disparo de eventos futuros. De acuerdo con el criterio establecido, se elaboró la Tabla 5, que relaciona las poblaciones que resultan según el orden de prioridades establecido, indicando a su vez las fallas principales que condicionarán la definición de los correspondientes escenarios de peligrosidad. En estas poblaciones y atendiendo a la prioridad establecida, se recomendó finalmente hacer un estudio con mayor detalle del riesgo, y definir escenarios particulares de daño para sismos concretos que puedan afectar a la población, con cierta localización y magnitud, con un enfoque determinista. De la Tabla 5 se deduce que la localidad de Lorca es una de las seis que resultaron con prioridad máxima para el desarrollo de estudios de riesgo sísmico a nivel local (Benito et al, 2005). 


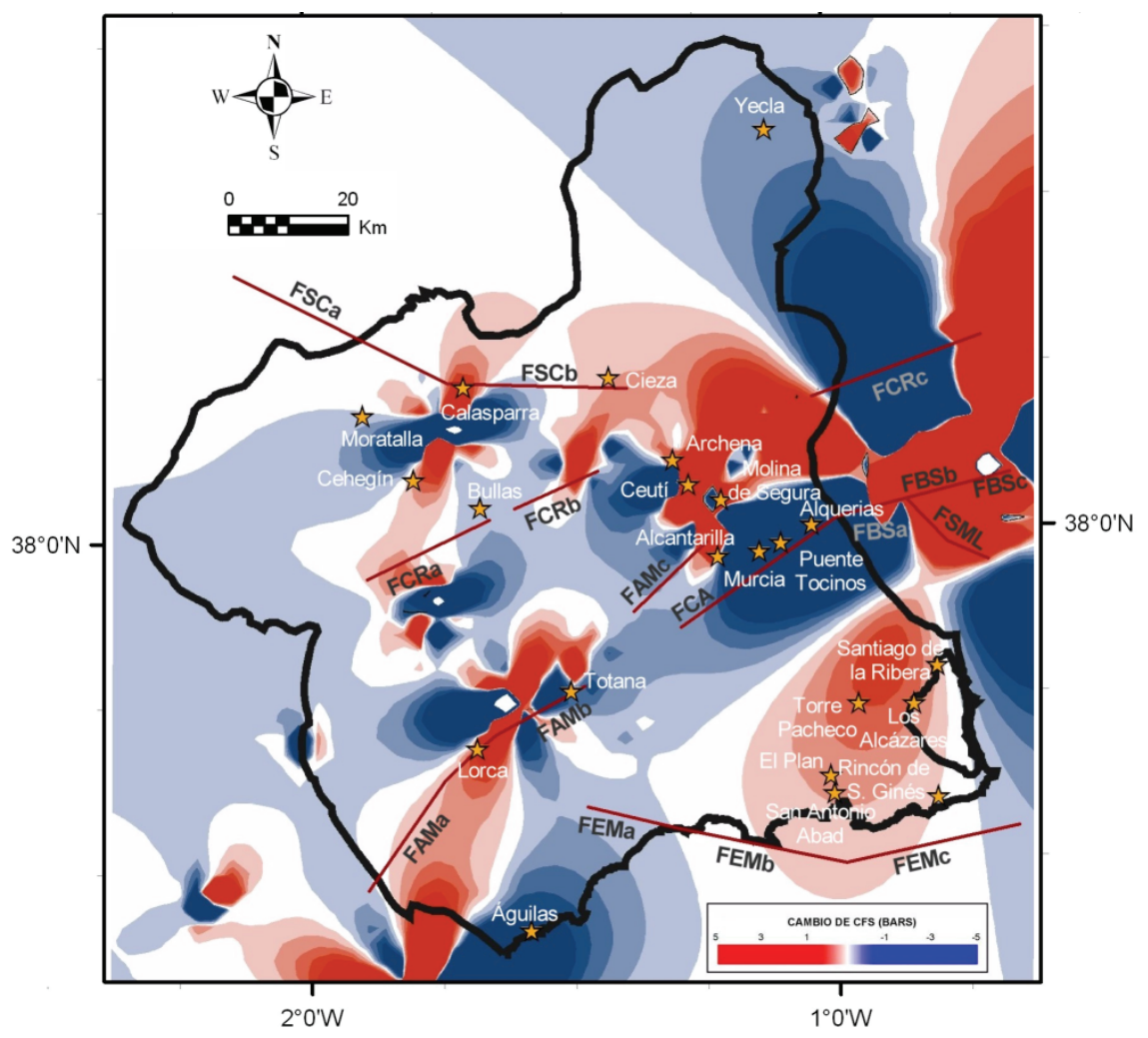

Fig. 14. Mapa final integrando distintos resultados del proyecto: zonas de máximo incremento de esfuerzos, resultantes del estudio de cambio de esfuerzos estáticos de Coulomb; principales fallas activas en la región resultantes del análisis sismotectónico y poblaciones que han resultado con mayor daño neto esperado para los movimientos con periodo de retorno de 475 años. Fallas principales (la letra indica el segmento de la falla). Fuente: RISMUR, Benito et al (2008): FAM: Falla de Alhama de Murcia: a:Pto. Lumbreras-Lorca, b:Lorca-Totana, c:Alhama-Alcantarilla; FS: Falla de Socovos-Calasparra: a:Central o Calasparra, b:Línea Eléctrica; FCR: Falla de Crevillente: a: Campo Coy-Bullas, b:Bullas$S^{a}$ Ricote, c:Abanilla-Vinalopó; FCA: Falla de Carrascoy; FBS: Falla del Bajo Segura: a: Hurchillos, b:Benejúzar, c:Guardamar; FSM: Falla de San Miguel de Salinas; FEM: Falla de Las Moreras-Escarpe de Mazarrón, a: $\mathrm{S}^{\mathrm{a}}$ Moreras, b: Pto.Mazarrón-C.Tiñoso, c:C.Tiñoso-C.Palos 
Tabla 5. Relación de poblaciones con mayor daño esperado y orden de prioridad establecido para la definición de escenarios particulares de peligrosidad y riesgo (ver mapa de la Figura 14). Se indican las fallas principales más próximas y las correspondientes distancias mínimas a los municipios respectivos.

\begin{tabular}{|c|c|c|c|c|c|}
\hline \multirow[b]{2}{*}{ ENTIDAD } & \multirow[b]{2}{*}{ MUNICIPIO } & \multicolumn{3}{|c|}{ FALLAS PRINCIPALES PRÓXIMAS } & \multirow{2}{*}{$\begin{array}{l}\text { Priori- } \\
\text { dad } \\
\text { (1 es } \\
\text { máximo) }\end{array}$} \\
\hline & & Falla & Segmento & $\begin{array}{l}\text { Distancia } \\
\text { mín (km) }\end{array}$ & \\
\hline \multirow[t]{2}{*}{ LORCA } & \multirow[t]{2}{*}{ Lorca } & \multirow{2}{*}{$\begin{array}{c}\text { Alhama de } \\
\text { Murcia }\end{array}$} & Lorca-Totana & 0 & \multirow[b]{2}{*}{1} \\
\hline & & & $\begin{array}{l}\text { Pto.Lumbreras- } \\
\text { Lorca }\end{array}$ & 2 & \\
\hline \multirow[t]{2}{*}{ CALASPARRA } & \multirow[t]{2}{*}{ Calasparra } & \multirow{2}{*}{$\begin{array}{c}\text { Socovos- } \\
\text { Calasparra }\end{array}$} & Línea eléctrica & 0 & \multirow{2}{*}{1} \\
\hline & & & Central & 3 & \\
\hline \multirow[t]{2}{*}{ ALCANTARILLA } & \multirow[t]{2}{*}{ Alcantarilla } & $\begin{array}{l}\text { Alhama de } \\
\text { Murcia }\end{array}$ & Alhama-Alcantarilla & 3 & \multirow[t]{2}{*}{1} \\
\hline & & Carrascoy & $\mathrm{S}^{\mathrm{a}}$ de Carrascoy & 6 & \\
\hline CIEZA & Cieza & $\begin{array}{c}\text { Socovos- } \\
\text { Calasparra }\end{array}$ & Línea eléctrica & 2 & 1 \\
\hline \multirow{2}{*}{$\begin{array}{l}\text { MOLINA DE } \\
\text { SEGURA }\end{array}$} & \multirow{2}{*}{$\begin{array}{l}\text { Molina de } \\
\text { Segura }\end{array}$} & Crevillente & Bullas-S ${ }^{\text {a }}$ Ricote & 8 & \multirow{2}{*}{1} \\
\hline & & Carrascoy & $\mathrm{S}^{\mathrm{a}}$ de Carrascoy & 13 & \\
\hline CEHEGIN & Cehegín & Crevillente & Campo Coy-Bullas & 12 & 1 \\
\hline \multirow[t]{2}{*}{ CEUTI } & \multirow[t]{2}{*}{ Ceutí } & $\begin{array}{l}\text { Alhama de } \\
\text { Murcia }\end{array}$ & Alhama-Alcantarilla & 10 & \multirow[t]{2}{*}{2} \\
\hline & & Crevillente & Bullas-S ${ }^{\text {a }}$ Ricote & 15 & \\
\hline \multirow[t]{2}{*}{ ARCHENA } & \multirow[t]{2}{*}{ Archena } & Crevillente & Bullas-S ${ }^{a}$ Ricote & 12 & \multirow[b]{2}{*}{2} \\
\hline & & $\begin{array}{l}\text { Alhama de } \\
\text { Murcia }\end{array}$ & Alhama-Alcantarilla & 15 & \\
\hline $\begin{array}{l}\text { RINCON DE SAN } \\
\text { GINES }\end{array}$ & Cartagena & $\begin{array}{l}\text { Escarpe de } \\
\text { Mazarrón }\end{array}$ & $\begin{array}{l}\text { Cabo Tiñoso-C.de } \\
\text { Palos }\end{array}$ & 8 & 2 \\
\hline $\begin{array}{l}\text { SAN ANTONIO } \\
\text { ABAD }\end{array}$ & Cartagena & $\begin{array}{l}\text { Escarpe de } \\
\text { Mazarrón }\end{array}$ & $\begin{array}{l}\text { Pto.Mazarrón- } \\
\text { C.Tiñoso }\end{array}$ & 11 & 2 \\
\hline EL PLAN & Cartagena & $\begin{array}{l}\text { Escarpe de } \\
\text { Mazarrón }\end{array}$ & $\begin{array}{l}\text { Pto.Mazarrón- } \\
\text { C.Tiñoso }\end{array}$ & 14 & 2 \\
\hline LOS ALCAZARES & Los Alcázares & - & - & - & 2 \\
\hline TORRE PACHECO & $\begin{array}{c}\text { Torre } \\
\text { Pacheco }\end{array}$ & - & - & - & 2 \\
\hline $\begin{array}{l}\text { SANTIAGO DE LA } \\
\text { RIBERA }\end{array}$ & San Javier & - & - & - & 2 \\
\hline TOTANA & Totana & $\begin{array}{l}\text { Alhama de } \\
\text { Murcia }\end{array}$ & Lorca-Totana & 0 & 3 \\
\hline BULLAS & Bullas & Crevillente & Campo Coy-Bullas & 3 & 3 \\
\hline \multirow[t]{3}{*}{ MURCIA } & \multirow[t]{3}{*}{ Murcia } & Carrascoy & $\mathrm{S}^{\mathrm{a}}$ de Carrascoy & 3 & \multirow{3}{*}{3} \\
\hline & & $\begin{array}{c}\text { Alhama de } \\
\text { Murcia }\end{array}$ & Alhama-Alcantarilla & 9 & \\
\hline & & Bajo Segura & Hurchillos & 15 & \\
\hline \multirow[t]{2}{*}{ ALQUERIAS } & Murcia & Carrascoy & $\mathrm{S}^{\mathrm{a}}$ de Carrascoy & 1 & 3 \\
\hline & & Bajo Segura & Hurchillos & 5 & 3 \\
\hline PUENTE & Murcia & Carrascoy & $\mathrm{S}^{\mathrm{a}}$ de Carrascoy & 2 & \\
\hline TOCINOS & & Bajo Segura & Hurchillos & 12 & 3 \\
\hline & & $\begin{array}{l}\text { Alhama de } \\
\text { Murcia }\end{array}$ & Alhama-Alcantarilla & 11 & 3 \\
\hline MORATALLA & Moratalla & Socovos- & Central & 11 & 3 \\
\hline & & Calasparra & Línea eléctrica & 15 & 3 \\
\hline YECLA & Yecla & - & - & - & 4 \\
\hline AGUILAS & Águilas & - & - & - & 4 \\
\hline
\end{tabular}




\subsection{Daños observados después del terremoto}

\subsubsection{Triaje técnico de edificios}

Desde el mismo instante del terremoto, un equipo de arquitectos y arquitectos técnicos voluntarios se encargaron del triaje, o evaluación de emergencia del parque inmobiliario de Lorca. El objeto del triaje fue una primera evaluación básica de seguridad y habitabilidad de los edificios, por lo que los datos debían tomarse como orientativos. Un total de 7839 edificios habían sido analizados por los arquitectos al finalizar la primera semana tras el sismo. Estos datos fueron incorporados a la planimetría municipal para disponer de datos de daños georreferenciados.

Los edificios se evaluaron asignando etiquetas de color verde, amarillo y rojo, cuyo grado de daño o estado del edificio se describe a continuación:

- La etiqueta verde significó seguridad de uso, si bien el edificio podía ostentar daños ligeros como fisuras, grietas o pequeños desprendimientos.

- La etiqueta amarilla implicaba edificio con daños y acceso al mismo condicionado a la eliminación del riesgo, por ejemplo mediante derribo de techos o parapetos dañados, o bien permitiendo su uso con precaución. Estos edificios podían tener además daños estructurales.

- La etiqueta roja significó edificio dañado estructuralmente, siendo necesaria la prohibición de acceso al inmueble. La mayoría de las etiquetas rojas emitidas se referían a edificios con daños a los pilares.

El mapa correspondiente a la distribución de daños según esta clasificación se presenta en la Figura 15. 


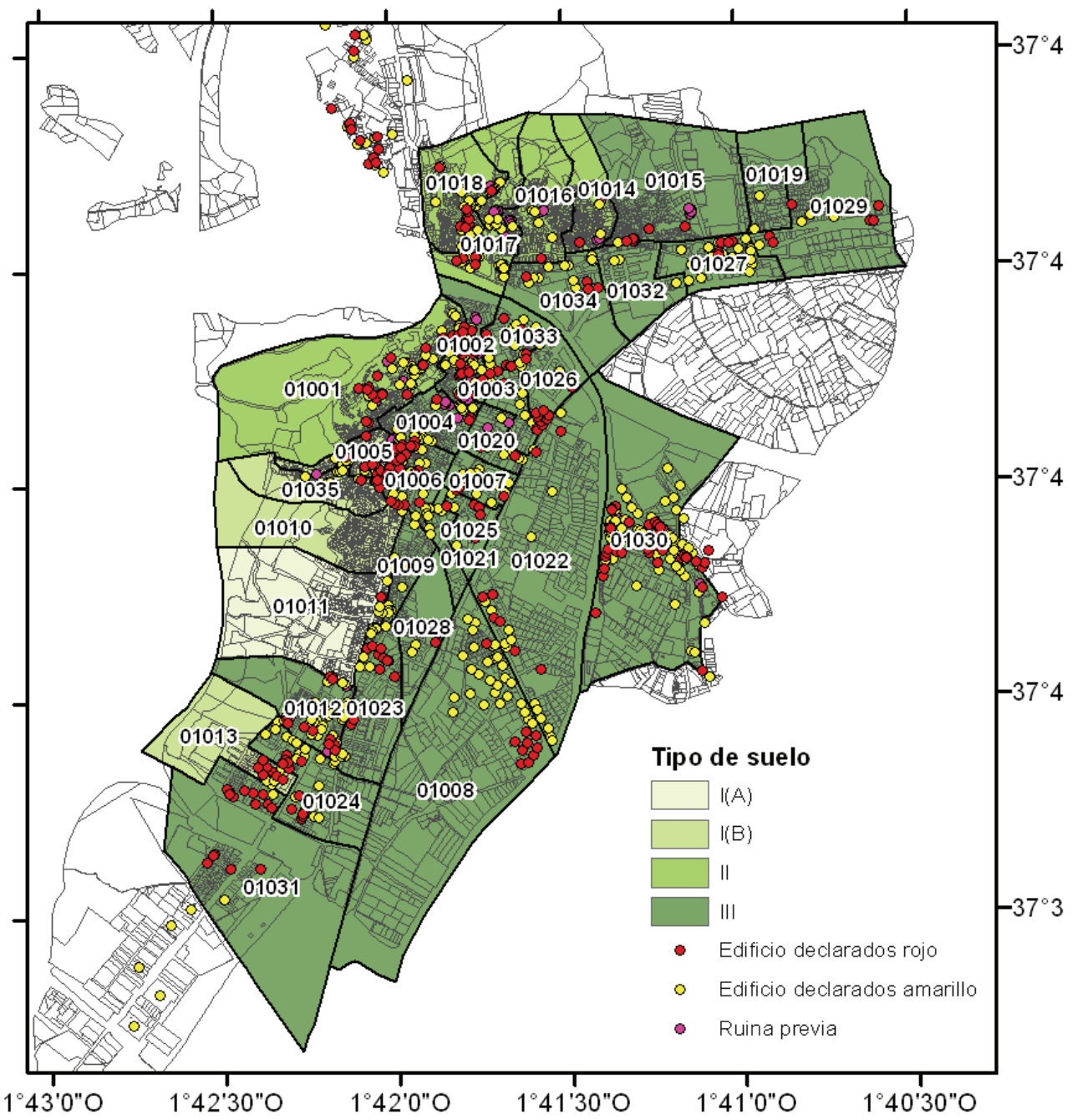

Fig. 15. Daños en Lorca indicando edificios declarados con etiqueta amarilla (daños moderados) y roja (daños graves) por distrito censal y tipo de suelo, elaborado con datos del 1 de junio de 2011. Se indican las ruinas previas al terremoto en color rosa.

\subsubsection{Datos de daños}

En el área municipal analizada con un total de 5155 edificios, 889 de ellos (el $13 \%$ del total) han obtenido la calificación de daño amarillo o rojo. Es llamativa la incidencia de daños en algunos distritos censales del barrio de la Viña, como 
son los números 1013 y 1024 con porcentajes de edificios dañados del $41 \%$ y $40 \%$ sobre el total de edificios de su distrito.

El distrito censal 1004, representativo del casco histórico, sufre daños amarillos o rojos en un $16 \%$ del total de edificios de su distrito.

$\mathrm{Al}$ igual que en el Barrio de la Viña, el barrio de la Avenida de las Fuerzas Armadas, representado por el distrito 1027, tiene daños en el $40 \%$ del total de edificios. Los datos pormenorizados por distrito censal se presentan en la Tabla 6.

El mapa de daños se ha superpuesto sobre las características de suelo del municipio de Lorca, realizado en el proyecto SISMOZON (Navarro et al., 2008), donde se caracterizan 4 tipos de suelos que varían de firme a blando, denominados IA; IB II y III.

\subsubsection{Análisis referentes al riesgo sísmico en Lorca y a los daños observados en 2011}

La ciudad de Lorca resultó con un riesgo relativamente alto en la provincia de Murcia, según los resultados del proyecto RISMUR, basado en datos de edificación de la campaña del Instituto Nacional de Estadística de 2001 (y adaptada a 2004). El daño neto se caracterizó mediante el número esperado de edificios inhabitables, que resultó ser de 663. Tras el terremoto de Lorca de 2011, si consideramos que, tanto los edificios marcados en rojo como una proporción de los marcados en amarillo (según el triaje realizado) son inhabitables, resultaría una cantidad de edificios de este tipo en Lorca del mismo orden de magnitud que los obtenidos en RISMUR. Esta cantidad dependería del porcentaje de edificios marcados en amarillo que se consideren inhabitables, oscilando entre 306 (si tomamos el $0 \%$ ) y 805 (tomando el $100 \%$ ). Un valor central sería de 556 edificios inhabitables, en caso de considerar el $50 \%$ de los etiquetados en amarillo. Conviene remarcar, no obstante, que en RISMUR no se simuló un determinado escenario sísmico, sino el daño acumulado por el movimiento esperado en 50 años, con un $10 \%$ de probabilidad de excedencia. Los resultados no son directamente comparables con el caso de Lorca, pero es de destacar que lo observado en el terremoto está dentro de lo previsible en ese periodo de 50 años.

Una comparación más directa puede hacerse con los resultados del proyecto SISMOZON, en el cual sí se simula un determinado escenario sísmico, concretamente el definido mediante un análisis de desagregación de la peligrosidad, del que resultó un terremoto de control dominante (para periodo de retorno de 475 años) caracterizado por un par $\mathrm{Mw}$ 4.5-5.0 y distancia 0-10 km. Este sismo presenta características muy similares a las que concurren en el sismo del 11 de mayo, en cuanto a magnitud y falla generadora, por lo que la comparación de los daños simulados en el citado escenario y los causados por el terremoto adquiere especial relevancia. Se realiza entonces una comparación entre los edificios dañados con triaje amarillo y rojo y aquellos modelizados en SISMOZON con daño moderado, extenso y colapso, ordenados por distritos censales. Los datos se presentan en la Tabla 6. 
Tabla 6. Comparación entre el número de edificios dañados en el terremoto de 2011 y el número previsto previamente según el estudio SISMOZON, distribuidos por distrito censal para la ciudad de Lorca. Ver figura 14. Los edificios dañados en el terremoto son los identificados en el triaje con los colores amarillo, rojo y negro. Los edificios dañados en nuestra modelización, son aquellos que han resultado con daño moderado, extensivo y completo (según la clasificación de daño RISK-UE).

\begin{tabular}{|c|c|c|c|c|c|}
\hline \multicolumn{2}{|c|}{ Edificios en Lorca } & \multicolumn{2}{|c|}{$\begin{array}{l}\text { Observado en } \\
\text { terremoto } 2011\end{array}$} & \multicolumn{2}{|c|}{$\begin{array}{l}\text { modelizado en } \\
\text { estudio previo }\end{array}$} \\
\hline distrito censal & Total Edificios & \begin{tabular}{|c|} 
Total \\
Dañados
\end{tabular} & $\%$ & \begin{tabular}{c|} 
Total \\
Dañados
\end{tabular} & $\%$ \\
\hline 1001 & 584 & 54 & $9,25 \%$ & 94 & $16,10 \%$ \\
\hline 1002 & 220 & 62 & $28,18 \%$ & 31 & $14,09 \%$ \\
\hline 1003 & 62 & 15 & $24,19 \%$ & 4 & $6,45 \%$ \\
\hline 1004 & 249 & 40 & $16,06 \%$ & 40 & $16,06 \%$ \\
\hline 1005 & 352 & 51 & $14,49 \%$ & 36 & $10,23 \%$ \\
\hline 1006 & 244 & 59 & $24,18 \%$ & 30 & $12,30 \%$ \\
\hline 1007 & 45 & 19 & $42,22 \%$ & 4 & $8,89 \%$ \\
\hline 1008 & 136 & 38 & $27,94 \%$ & 19 & $13,97 \%$ \\
\hline 1009 & 143 & 15 & $10,49 \%$ & 14 & $9,79 \%$ \\
\hline 1010 & 390 & 5 & $1,28 \%$ & 45 & $11,54 \%$ \\
\hline 1011 & 369 & 23 & $6,23 \%$ & 33 & $8,94 \%$ \\
\hline 1012 & 208 & 44 & $21,15 \%$ & 26 & $12,50 \%$ \\
\hline 1013 & 46 & 17 & $36,96 \%$ & 2 & $4,35 \%$ \\
\hline 1014 & 344 & 1 & $0,29 \%$ & 42 & $12,21 \%$ \\
\hline 1015 & 410 & 8 & $1,95 \%$ & 63 & $15,37 \%$ \\
\hline 1016 & 361 & 8 & $2,22 \%$ & 39 & $10,80 \%$ \\
\hline 1017 & 325 & 27 & $8,31 \%$ & 32 & $9,85 \%$ \\
\hline 1018 & 399 & 35 & $8,77 \%$ & 42 & $10,53 \%$ \\
\hline 1019 & 75 & 8 & $10,67 \%$ & 2 & $2,67 \%$ \\
\hline 1020 & 51 & 11 & $21,57 \%$ & 5 & $9,80 \%$ \\
\hline 1021 & 32 & 8 & $25,00 \%$ & 1 & $3,13 \%$ \\
\hline 1022 & 202 & 23 & $11,39 \%$ & 27 & $13,37 \%$ \\
\hline 1023 & 116 & 27 & $23,28 \%$ & 10 & $8,62 \%$ \\
\hline 1024 & 81 & 30 & $37,04 \%$ & 6 & $7,41 \%$ \\
\hline 1025 & 38 & 8 & $21,05 \%$ & 2 & $5,26 \%$ \\
\hline 1026 & 57 & 13 & $22,81 \%$ & 5 & $8,77 \%$ \\
\hline 1027 & 70 & 28 & $40,00 \%$ & 3 & $4,29 \%$ \\
\hline 1028 & 61 & 8 & $13,11 \%$ & 6 & $9,84 \%$ \\
\hline 1029 & 51 & 10 & $19,61 \%$ & 3 & $5,88 \%$ \\
\hline 1030 & 334 & 102 & $30,54 \%$ & 50 & $14,97 \%$ \\
\hline 1031 & 215 & 21 & $9,77 \%$ & 36 & $16,74 \%$ \\
\hline 1032 & 74 & 6 & $8,11 \%$ & 11 & $14,86 \%$ \\
\hline 1033 & 71 & 22 & $30,99 \%$ & 8 & $11,27 \%$ \\
\hline 1034 & 83 & 13 & $15,66 \%$ & 8 & $9,64 \%$ \\
\hline 1035 & 264 & 17 & $6,44 \%$ & 27 & $10,23 \%$ \\
\hline Total & 6762 & 876 & $12,95 \%$ & 806 & $11,92 \%$ \\
\hline
\end{tabular}


Los resultados son similares en cuanto al total de edificios dañados, sin embargo son muy dispares en cuanto a su distribución geográfica. El estudio previo no detectó la gran vulnerabilidad de los distritos fuertemente dañados, como es el caso de la avenida de las Fuerzas Armadas (1027). Este distrito resultó dañado en un $40 \%$ del total de sus edificios frente a la estimación de $4,29 \%$ que arrojaba el estudio previo. De forma similar, el distrito 1013 del barrio de La Viña quedó dañado en un $36 \%$ frente al $4,35 \%$ que preveía el estudio.

Por otra parte, el distrito 1016, correspondiente a zonas de edificación tradicional de baja altura, quedó dañado en un 2,22\% frente al 10,80\% que preveía el estudio.

La comparación evidencia que las curvas de fragilidad utilizadas en estudios de riesgo son conservadoras para la edificación tradicional y liberales para la edificación tecnológica, prediciendo en el caso de estas últimas daños muy inferiores a los observados realmente. Las bajas estimaciones de daño que preveía el estudio en estos distritos se debe, de hecho, a la mayor incidencia de edificación moderna y tecnológica.

\section{Discusión final y lecciones aprendidas}

El terremoto de Lorca no ha resultado una sorpresa, ni por su localización, ni por su magnitud. La falla de Alhama de Murcia, fuente del terremoto, es una de las más activas del Sureste de España, y un sismo de magnitud $\mathrm{M}_{\mathrm{w}} 5.1$ no es el más grande que cabe esperar en esta falla. Lo sorprendente ha sido el grado de daño causado, que puede explicarse por la alta aceleración pico registrada, de $0.37 \mathrm{~g}$, además de por las características del parque inmobiliario de la zona.

Desde el punto de vista sismológico, cabe preguntarse si esa aceleración es anormalmente alta para un evento de magnitud 5.1. Varias consideraciones son necesarias al respecto: el foco fue muy superficial, con una profundidad de unos 3 $\mathrm{km}$ y ese valor pico fue registrado en la estación de Lorca, a $3 \mathrm{~km}$ de distancia epicentral y situada justo en el plano de ruptura. Fuera de este plano, la aceleración experimenta una fuerte atenuación. De hecho, en la siguiente estación (situada a $24 \mathrm{~km}$ del epicentro) el valor registrado fue de $0,02 \mathrm{~g}$, casi 20 veces inferior al anterior.

$\mathrm{Al}$ igual que se ha evidenciado en otros sismos recientes (p.e L'Aquila, 2009), las aceleraciones se disparan en el plano de ruptura, cayendo bruscamente fuera del mismo. Parece existir un "efecto fuente" que en otros terremotos recientes no hemos podido comprobar, al no disponer de estaciones tan próximas. Si asumimos dicho efecto, la aceleración no resulta tan sorprendente. El problema es que, en este caso, la población está situada justo sobre la falla de Alhama de Murcia y se ha visto directamente afectada por ese efecto de la ruptura. Seguramente a distancias superiores a $10 \mathrm{~km}$ el movimiento habrá sido mucho menor y si la población hubiera estado a esa distancia de la falla no habría sufrido el mismo grado de daño. Esta es una de las lecciones aprendidas del terremoto, que nos conduce a poner especial atención hacia poblaciones que se encuentren en una situación similar a Lorca, ubicadas en la vecindad de fallas activas. Para minimi- 
zar el riesgo en estas poblaciones, debería considerarse el citado efecto fuente en el diseño sismorresistente de sus edificaciones.

Por lo demás, el terremoto entraba dentro de lo esperado, según las conclusiones del proyecto RISMUR, dirigido al estudio del riesgo sísmico de la región de Murcia para el plan de emergencias SISMIMUR, activado precisamente con el terremoto del 11 de mayo de 2011.

Una de ellas fue destacar Lorca como población prioritaria sobre la que desarrollar planes de emergencia a nivel municipal, dada la confluencia de los siguientes factores: alto riesgo esperado, proximidad a una falla activa y situación dentro de una zona cargada de esfuerzos de Coulomb como resultado de la sismicidad previa (Benito et al. 2008).

También se puso de manifiesto que los terremotos de magnitud entorno a 5.0, a corta distancia epicentral, presentan elevada contribución a la peligrosidad para periodo de retorno de 475 años, es decir para probabilidades de $10 \%$ en 50 años, que son las consideradas en diseño de viviendas convencionales. Esta fue otra conclusión del proyecto RISMUR, que también se evidenció en el estudio de riesgo de Andalucía (proyecto SISMOSAN). Todo ello reclama nuestra atención hacia los terremotos de magnitud moderada, que además de ser los que más contribuyen a la peligrosidad para la probabilidad citada en el sur y sureste peninsular, pueden generar elevadas aceleraciones en la proximidad de la fuente y por tanto causar importantes daños en poblaciones ubicadas en el entorno. Esta importante lección debería tenerse en cuenta en próximas ediciones de la normativa sismorresistente de nuestro país.

Por último, cabe proponer algunas recomendaciones como consecuencia de las lecciones aprendidas en el terremoto de Lorca de 2011 y en los anteriores de 1999, 2002 y 2005, que deberían considerarse en la revisión de la normativa, destacando especialmente:

- Necesidad de modelizar las fallas activas e incluirlas como unidades independientes en el cálculo de la peligrosidad, a fin de obtener aceleraciones más realistas en la proximidad de dichas fallas.

- Necesidad de ajustar las formas espectrales de la normativa con las deducidas de los registros reales.

- Necesidad de determinar posibles efectos de resonancia entre el comportamiento dinámico del suelo y el de las estructuras.

- Incorporación a la norma sismorresistente de previsiones claras para los elementos no estructurales de la edificación tecnológica actual.

- Necesidad de calibrar curvas de fragilidad para mejorar la predicción de daños en estructuras tecnológicas, teniendo en cuenta además el daño previsible por elementos no estructurales. 


\section{Referencias bibliográficas}

ABRAHAMSON, N. A. \& SILVA, W. J. (2008). Abrahamson \& Silva NGA ground motion relations for the geometric mean horizontal component of peak and spectral ground motion parameters. PEER Report Draft v2, Pacific Earthquake Engineering Research Center, Berkeley, CA, pp 380.

AKKAR, S. \& BOOMER, J.J. (2010). Empirical Equations for the Prediction of PGA, PGV, and Spectral Accelerations in Europe, the Mediterranean Region, and the Middle East. Seismol. Res. Lett., vol. 81, 195-206.

BADAL, J., SAMARDJIEVA, E. \& PAYO, G. (2000). Moment magnitudes for early (1923-1961) instrumental Iberian earthquakes" Bull. Seism. Soc. Am. 90, 1161-1173.

BENITO, B., MURPHY, P., TSIGE, M., MARTÍNEZ-DÍAZ, J. J., GASPARESCRIBANO, J. M., GARCÍA-MAYORDOMO, J., JIMÉNEZ, M. E., GARCÍA, M. J., CANORA, C., ÁLVAREZ-GÓMEZ, J. A. \& GARCÍAFLORES, I. (2006). Riesgo Sísmico en la Región de Murcia: Proyecto RISMUR. Informe final, 7 volúmenes y un anexo, Instituto Geográfico Nacional, Protección Civil Región de Murcia.

BENITO, B., CAPOTE, B., MURPHY, P., GASPAR-ESCRIBANO, J. M., MARTÍNEZ-DÍAZ, J. J., TSIGE, M., STICH, D., GARCÍA-MAYORDOMO, J., GARCÍA, M. J., JIMÉNEZ, M. E., INSUA-ARÉVALO, J. M., ÁLVAREZGÓMEZ, J. A. \& CANORA, C. (2007). An overview of the damaging and low magnitude La Paca earthquake (Mw 4.8) on January 29th, 2005. Context; seismotectonics; and seismic risk implications for South East Spain, Bull. Seism. Soc. Am., 97, 671-690.

BENITO, B., GASPAR-ESCRIBANO, J. M., MARTÍNEZ-DÍAZ, J. J., GARCÍA RODRÍGUEZ, M. J., JIMÉNEZ PEÑA, E., CANORA, C. \& ÁLVAREZGÓMEZ, J.A. (2008). The RISMUR Project: Seismic Risk assessment of the Murcia province (SE Spain). Boll.Geofis.Teor. Applic., 49, 3-15.

BUFORN, E. \& SANZ DE GALDEANO, C. (2001). Focal mechanism of Mula (Murcia, Spain) earthquake of February 2, 1999. J. Seismol., 5, 277-280.

BUFORN, E., BENITO, B. SANZ DE GALDEANO, C., FRESNO, C. DEL, MUÑOZ, D. \& RODRÍGUEZ, I. (2005). Study of the damaging earthquakes of 1911, 1999 and 2002 in risk implications Bull. Seismol. Soc. Am., 95, 549567.

CAMPBELL, K.W. \& BOZORGNIA, Y. (2008). Campbell-Bozorgnia NGA ground motion relations for the geometric mean horizontal component of peak and spectra ground motion parameters. PEER Report 2007/02,Pacific Earthquake Engineering Research Center, Berkeley, CA, pp 240.

CHIOU B. \& YOUNGS R.R. (2008). Chiou-Youngs PEER-NGA empirical ground motion model for the average horizontal component of peak acceleration and pseudo-spectral acceleration for spectral periods of 0.01 to $10 \mathrm{sec}$ - 
onds. PEER Report Draft, Pacific Earthquake Engineering Research Center, Berkeley, CA, $219 \mathrm{pp}$

CRESPO, M.J. (2011). Análisis de la peligrosidad sísmica en la Península Ibérica con un método basado en estimadores de densidad Kernel. Tesis doctoral. E.T.S.I. Caminos, Canales y Puertos (UPM).

GARCÍA-BLANCO, R.M. (2009). Caracterización del potencial sísmico y su influencia en la determinación de la peligrosidad sísmica probabilística. Tesis doctoral. E.T.S.I. Minas. (UPM).

GARCÍA-MAYORDOMO, J. (2005). Caracterización y análisis de la peligrosidad sísmica en el sureste de España, Tesis doctoral. Universidad Complutense de Madrid..

GARCÍA-MAYORDOMO, J. GASPAR-ESCRIBANO, J. M. \& BENITO, B. (2007). Seismic Hazard Assessment of the Province of Murcia (SE Spain): Analysis of Source Contribution to Hazard. J. Seismol. 11, 453-471.

GASPAR-ESCRIBANO, J. M., BENITO, B. \& GARCÍA-MAYORDOMO, J. (2008). Hazard-Consistent Response Spectra in the Region of Murcia (SE Spain). Bull. Earthquake Eng. 6, 179-196.

GASPAR-ESCRIBANO, J. M. \& BENITO, B. (2007). Ground motion characterisation of low-to-moderate seismicity zones and implications for seismic design: lessons from recent, Mw 4.8, damaging earthquakes in SE Spain. Bull. Seismol. Soc. Am., 97, 531-544.

IGN (1999). Serie sísmica de Mula (Murcia). Segundo Informe general. Subdirección General de Geodesia y Geofísica, Madrid, $35 \mathrm{pp}$

LOPEZ-COMINO, J. A., MANCILLA, F., MORALES, J. \& STICH D. (2012) Rupture directivity of the 2011, Mw 5.2 Lorca earthquake (Spain). Geophysical Research Letters, 39 p. L03301

MANCILLA, F. L., AMMON, C., HERRMANN, R. B.\& MORALES, J. (2002). Faulting Parameters of the 1999 Mula Earthquake, Southeastern Spain. Tectonophysiscs, 354, 139-155.

MARTÍNEZ SOLARES, J. M. \& MÉZCUA, J. (2002). Catálogo sísmico de la Península Ibérica (880 a. C.-1900). Monografía 18, Instituto Geográfico Nacional, Madrid, $253 \mathrm{pp}$.

MARTÍNEZ-DÍAZ, J. J., RIGO, A., LOUIS, L., CAPOTE, R., HERNÁNDEZENRILE, J. L., CARREÑO, E. \& TSIGE, M. (2002). Caracterización geológica y sismotectónica del terremoto de Mula (febrero de 1999, Mb: 4,8) mediante la utilización de datos geológicos, sismológicos y de interferometría de RADAR (INSAR). Boletín Geológico y Minero, 113, 23-33.

MARTÍNEZ-DÍAZ, J.J., BEJAR-PIZARRO, M., ÁLVAREZ-GÓMEZ, J.A., MANCILLA, F.L., STICH, D., HERRERA, G. \& MORALES, J. (2012). Tectonic and seismic implications of an intersegment rupture. The damaging May 11th 2011 Mw 5.2 Lorca, Spain, earthquake. Tectonophysics. 546-547, 28-37. 
MEZCUA, J. \& MARTÍNEZ SOLARES, J. M. (1983). Sismicidad del área Ibero-Mogrebi. Instituto Geográfico Nacional, Pub. 203, 301 pp.

NAVARRO, M., GARCÍA-JEREZ, JA., ALCALÁ, FJ., VIDAL, F., ENOMOTO, T., LUZÓN, F. \& CREUS, C. (2008). VS30 Structure of Lorca town (SE Spain) from Ambient Noise Array Observations. 31st General Assembly of the European Seismological Commission ESC 2008. Hersonissos, Crete, Greece, 7-12 September.

NORMA DE LA CONSTRUCCIÓN SISMORRESISTENTE ESPAÑOLA (NCSE-02) (2002). Real Decreto 997/2002, de 27 de septiembre, por el que se aprueba la norma de construcción sismorresistente: parte general y edificación (NCSR-02). Boletín Oficial del Estado, vol. 244, 35898-35967.

REY PASTOR, A. (1949). Comarca sísmica de Caravaca y el sismo del 23 de junio de 1948. Instituto Geográfico y Catastral, Madrid, 21-33. 